\title{
Numerical Simulations of Shock/Plume Interaction Using Structured Overset Grids
}

\author{
Jeffrey A. Housman*1 and Cetin C. Kiris ${ }^{\dagger 1}$ \\ ${ }^{1}$ NASA Ames Research Center, M/S 258-2, Moffett Field, CA 94035
}

\begin{abstract}
Computational simulations using structured overset grids with the Launch Ascent and Vehicle Aerodynamics (LAVA) solver framework are presented for predicting oblique shock/plume interaction effects to near-field sonic boom signatures. Standard second-order accurate as well as higher-resolution numerical discretizations are utilized and compared in the study. The numerical approach is compared with supersonic wind-tunnel data for three cases. The cases include an empty wind-tunnel at the operating conditions, an isolated shockgenerating diamond wedge within the tunnel, and a nozzle with diamond wedge configuration at five different nozzle pressure ratios. Solution sensitivity to numerical discretization is analyzed. Favorable comparisons between the computational results and experimental data of near-field pressure signatures are obtained. A simple prediction method for plume induced shock deflection is developed and results are compared with the CFD data.
\end{abstract}

CFD Computational Fluid Dynamics

$d p /$ pref $\quad$ (Pressure $\left.-P_{\text {ref }}\right) / P_{\text {ref }}$

LAVA Launch Ascent and Vehicle Aerodynamics solver framework

LES Large Eddy Simulation

MUSCL Monotonic Upstream-Centered Scheme for Conservation Laws

NPR Nozzle Pressure Ratio

PIV Particle Image Velocimetry

RANS Reynolds Averaged Navier-Stokes

SA Spalart-Allmaras Turbulence Model

SWT Supersonic Wind-Tunnel

WENO Weighted Essentially Non-Oscillatory

$\gamma \quad$ ratio of specific heats

$\beta \quad$ Oblique shock angle

$\mu \quad$ Mach angle

$-\rho \overline{u^{\prime} v^{\prime}} \quad$ Reynolds Averaged Shear Stress ( $x y$-component)

$\mu_{T} \quad$ Turbulent Eddy Viscosity

$\bar{S}_{x y} \quad$ Reynolds Averaged Strain Rate Tensor ( $x y$-component)

\section{Introduction}

$\mathrm{T}$

HE ground level noise generated by the sonic boom of an aircraft traveling faster than the speed of sound is a key barrier in commercially viable civilian supersonic transports. Computational Fluid Dynamics (CFD) is taking a lead role in the initial design and analysis of low boom aircraft concepts. As conceptual designs mature towards a flight ready model, exhaust plume and engine installation effects must be considered. Exclusion of these effects may be detrimental to the accuracy of the predicted near-field pressure signature towards the aft of the vehicle. Errors in this region may cause erroneous far-field boom propagation estimates

*Research Scientist, Applied Modeling and Simulation Branch, NAS Division, MS N258-2, AIAA Senior Member

${ }^{\dagger}$ Branch Chief, Applied Modeling and Simulation Branch, NAS Division, MS N258-2, AIAA Senior Member 
and lead to the selection of aircraft designs which may not realize a true low boom signature during flight. A detailed validation study is performed to assess the current CFD predictive capabilities of the Launch Ascent and Vehicle Aerodynamics (LAVA) solver framework ${ }^{1}$ for oblique shock/plume interaction.

An experimental investigation of an oblique shock wave interacting with a high-speed jet plume has recently been performed in the $1 \mathrm{ft} . \times 1 \mathrm{ft}$. Supersonic Wind-Tunnel (SWT) at NASA Glenn Research Center. $^{2}$ Measurements of static pressure signatures, Schlieren photography, and Particle Image Velocimetry (PIV) have been obtained. The data from this experiment is used to validate the present CFD methodology. Results for an empty tunnel, an isolated diamond wedge in the tunnel, and a 1.7 area ratio nozzle installed upstream of the diamond wedge and operating at five different nozzle pressure ratios (NPRs) are included in the study. CFD played a companion role during the experiment, whereby the results found flaws in the geometry and highlighted questionable measurement techniques.

An oblique shock interacting with a turbulent shear layer has been studied previously using LES. ${ }^{3}$ In the LES work the effects of the shock on the turbulent characteristics of the shear layer were identified. Numerical studies of oblique shock/plume interaction using both inviscid and RANS analyses have been reported recently, ${ }^{4,5}$ in which an in-depth code-to-code comparison was performed. In the present work, focus is placed on proper overset grid generation for accurate CFD prediction of oblique shock/plume interaction using RANS analysis, and investigating the physical mechanism of oblique shock deflection as the shock travels across a supersonic jet. Sensitivity of the shock deflection angle with respect to the fully-expanded jet Mach number is examined, and a simple prediction method for oblique shock deflection is developed. Due to the close proximity of the tunnel walls to the model, it is shown that viscous analysis is necessary to obtain the correct flow condition upstream of the test-section. In addition, proper boundary layer thickness along the tunnel walls was crucial to predict the angle of the reflected shocks from the tunnel walls, which experience shock/boundary layer interaction effects.

In Section II the computational methodology is described, including a brief description of the numerical discretization methods utilized in the study. The computed results are presented in Section III including the geometric model, the overset grid system, and three validation cases. Section IV describes the deflection effects of the jet on oblique shock waves.

\section{Computational Methodology}

The Launch Ascent and Vehicle Aerodynamics (LAVA) solver framework ${ }^{1}$ was utilized for the computational study. LAVA offers highly flexible meshing options and was developed with the intent of modeling highly complex geometry. The framework supports Cartesian and curvilinear structured grids as well as unstructured arbitrary polyhedral meshes. Overset grid technology ${ }^{6,7}$ is used to couple the solutions across the different mesh types. In this study, the curvilinear structured overlapping grid methodology is applied. The advantage of this approach for viscous analysis of sonic-boom related applications include: the ease of generating highly anisotropic grids to capture boundary layers, shocks, plume shear-layers, and wakes; the high efficiency and low memory footprint of the corresponding numerical algorithm; and the straightforward and inexpensive extension to higher-resolution low-dissipation convective flux discretizations. Allowing the structured grids to overlap each other not only simplifies the process of generating the grids (in comparison to structured multi-block abutting grids), it also allows for high quality meshes and an incremental approach to adding geometric components to the problem domain. Best practices on overset mesh generation ${ }^{8}$ are expanded upon for sonic-boom specific applications in this work. Validation of the current methodology for sonic-boom problems excluding plumes has been reported at the First AIAA Sonic Boom Prediction Workshop ${ }^{9}$ and companion paper. ${ }^{10}$

The compressible Reynolds Averaged Navier-Stokes (RANS) equations are solved using a finite-difference formulation applied to the transformed system of equations in strong conservation law form. ${ }^{11}$ The SpalartAllmaras (SA) ${ }^{12}$ turbulence model is used to close the system of equations. A modified Roe flux difference splitting scheme is used to discretize the convective fluxes. ${ }^{13,14}$ The left and right states of the approximate Riemann solver are reconstructed using either the MUSCL ${ }^{15}$ or the WENO ${ }^{16}$ approaches. For the WENO approach, a fifth-order accurate reconstruction in the coordinate direction is used, but the overall accuracy of the scheme remains second-order, since the metric terms and flux discretizations remain secondorder. ${ }^{17}$ The higher-order reconstruction reduces the overall numerical dissipation of the scheme, which will be demonstrated in the validation study. For the diffusive fluxes, standard second-order accurate central differencing is used. The convective terms in the SA turbulence model are discretized with a simple first- 
order upwind method, which is the suggested implementation of the model proposed in the original paper. ${ }^{12}$ The discretized equations are marched in pseudo-time to a steady-state solution. At each nonlinear-step, the system of equations is linearized and an alternating line-Jacobi relaxation procedure is applied. Local time-stepping with a $C F L=100$ is used to accelerate convergence. Domain decomposition and the Message Passing Interface (MPI) are used to enable a scalable parallel algorithm.

\section{Computed Results}

Computations were performed to investigate differences between inviscid analysis, viscous analysis, and wind-tunnel static pressure data, and to analyze plume deflection effects on the oblique shock angle. RANS and inviscid CFD analysis using the LAVA code are compared with the $1 \mathrm{ft} . \times 1 \mathrm{ft}$. SWT data for the empty tunnel, an isolated diamond wedge in the tunnel, and a 1.7 area ratio nozzle installed upstream of the diamond wedge and operating at NPR $=6,8,10,12$, and 14 .

The reference conditions used for the analysis are Mach 1.96 flow entering the tunnel test-section with a Reynolds number of 10.69 million per meter, a temperature of $168.9 \mathrm{~K}$, and pressure of $11.6 \mathrm{kPa}$ (1.68 psia). At the tunnel inlet, the stagnation temperature is set to $298.3 \mathrm{~K}$ with a stagnation pressure of $85.15 \mathrm{kPa}$ (12.35 psia). A cold jet is generated by the nozzle with a stagnation temperature of $294.4 \mathrm{~K}$ and stagnation pressures of $69.5,92.7,115.8,139.0$, and $162.1 \mathrm{kPa}$ corresponding to the five NPRs.

\section{III.A. Geometric Model}

The geometric model for the $1 \mathrm{ft}$. $\times 1 \mathrm{ft}$. SWT contains a 12.2 inch by 12 inch straight test section. The tunnel starts as a 12 inch by 12 inch square section that contours into a converging/diverging section ending in the same 12 inch by 12 inch square section 15.5 inches upstream of the start of the test-section. From the end of the diverging section, a linear taper in height from 12 inches to 12.2 inches is used. The overall length of the tunnel considered in this study is approximately 200 inches. A side-view of the tunnel is shown in Figure 1. Inside the tunnel, a 1.5 inch chord, $5^{\circ}$ half-angle diamond wedge is installed with a triangular support swept downstream at $25^{\circ}$. A side-view and isometric view of the diamond wedge is shown in Figure 2. The span of the diamond wedge is 3.6 inches and the height is 0.128 inch. Upstream of the wedge, a 1.7 area ratio nozzle is installed on a vertically extruded hexagonal support. The nozzle has an exterior length of $8.56 \mathrm{inch}$, an interior length of $4.43 \mathrm{inch}$, an outer diameter of 1 inch upstream of a 5 degree boat-tail section, and an inner exit diameter of 0.88 inch. Details of the nozzle geometry are displayed in Figure 3. A diagram of the complete installed configuration is shown in Figure 4.

\section{III.B. Structured Overset Grid System}

A structured overlapping grid system consisting of 27 zones and 108.5 million grid points was generated for a half-body representation of the complete configuration. All grids were generated using the Chimera Grid Tools ${ }^{18}$ software package version 2.1p (CGT v2.1p). Triple fringe layers are used to ensure proper overset communication when using the fifth-order WENO reconstruction, and strict overlap (i.e. no orphan points) is maintained throughout the entire grid system. The computed wall $y^{+}$is between one-quarter and one everywhere, which required smaller wall spacing on the nozzle interior walls due to the higher flow speeds. The grid system was build incrementally starting with the empty SWT which contains 49 million grid points. Figures 5 (a) and (b) show two views of the structured overlapping grid system generated for the tunnel. A body-wrapping grid is used to cover the surface of the tunnel walls, and grows hyperbolically to fill the tunnel interior next to the wall. The remaining SWT interior is filled with a deformed Cartesian box which overlaps the grid generated from the tunnel wall.

Next the grid system for the diamond wedge is created along with a highly refined wake grid downstream of the trailing edge. To begin, a 2D curve representation of the wedge is discretized with tight grid clustering at the leading and trailing edges, as well as the apexes of the diamond wedge. A 2D slice of the volume grid is grown hyperbolically from the curve, then redistributed again in the stream-wise direction to preserve the grid clustering at the leading and trailing edges. The 2D slice is then extrapolated in the span-wise direction with light clustering at the symmetry plane and dense clustering at the tip of the wedge. A cap grid is then generated to close the diamond wedge at the tip. Figure 6 (a) shows an image of the diamond wedge grid system including the surface grid, a 2D slice at the symmetry plane, and a slice of the cap grid. In order to capture the large gradient shear layers generated at the trailing edge of the diamond wedge, a highly 
refined anisotropic wake mesh was generated. The wake grid starts slightly upstream of the trailing edge of the diamond wedge (overlapping) and takes over from the wedge grid system close to the trailing edge. The tight cluster in the wake grid, matches the spacing of the diamond wedge grid at the trailing edge, then slowly fans out with stream-wise distance. Figure 6 (b) shows a close-up view of the transition from the diamond wedge grid to the wake grid at the symmetry plane. A total of 13.7 million grid points is used for the diamond wedge and wake grids.

The nozzle grid is built in a similar fashion. A curve is generated which represents the axisymmetric profile of the nozzle. Grid clustering is used at the nozzle throat, the nozzle exit, the expansion corner on the exterior of the nozzle, and the nose cone at the front of the nozzle. The smallest spacing is used at the nozzle exit, anticipating the large gradients from the plume shear layer. Once the curve has been discretized it is split into three overlapping sections; the nozzle interior, the nozzle lip, and the nozzle exterior. A 2D slice of the grid system is generated by hyperbolically marching from the walls of the three overlapping sections. The sections were split so that a different marching distance can be used for the interior (smaller) and the exterior (larger). The slice is then revolved around the stream-wise center of rotation to form a volume grid. A cap grid is generated at the nose, to remove the singular axis, and a deformed Cartesian box is generated in the nozzle interior to fill the remaining volume. Figure 7 (a) shows the nozzle interior surface grid along with a slice of the overlapping grid near the nozzle exit. While revolving the 2D slice, clustering was added near the bottom of the nozzle to properly communicate with the nozzle support strut grid, as shown in Figure 7 (b). To complete the nozzle grid system, an anisotropic cylindrical plume grid is created downstream of the nozzle exit to capture the shear layers of the plume. The singular axis of the plume grid is removed with a distorted Cartesian grid. Figure 8 displays a slice of the nozzle grid system on the symmetry plane near the nozzle exit, illustrating the transition from the nozzle lip grid to the plume grid. A total of 29.2 million grid points are used for the nozzle and plume grids.

Finally, overset grids for the nozzle and diamond wedge support struts are generated along with a refined wake grid which extends from the nozzle strut through the diamond wedge strut and ends at the same stream-wise location as the diamond wedge wake and nozzle plume grids. A total of 16.6 million grid points are used for the support struts and their wake grids. Figure 9 (a) shows a isometric view of the installed geometry shaded in gray (side and top wall of tunnel are blanked) along with the grids on the symmetry plane of the model. Figure 9 (b) shows the surface grids on the model along with a stream-wise slice of the grid system illustrating the span-wise extend of the anisotropic plume and wake grids. Close up views of the overlapping grid system on the symmetry plane are displayed in Figures 9 (c) and (d) showing the complex hole cutting of different grids in the overlap regions. In the case with the isolated diamond wedge in the tunnel, a subset of the wake grid from the nozzle strut was used to capture the wake from the diamond wedge strut.

\section{III.C. Case 1: Empty Tunnel}

The overset grid methodology was first applied to the empty SWT. Both RANS and inviscid analysis using second-order MUSCL reconstruction were performed, and comparison to two different probe types used to aquire the experimental data are presented. Figure 10 shows a contour plot of Mach number from the RANS result on the symmetry plane of the tunnel along with a close-up view of the diverging section with characteristic lines. Weak oblique shocks are created in the diverging section of the tunnel and travel downstream along the characteristic lines throughout the tunnel test section. These weak shocks generate small flow non-uniformities which must be resolved in order to capture the correct pressure signatures for the full configuration. Growth of the boundary layer along the tunnel walls is also observed. Since the cross-sectional area of the tunnel is relatively small compared to the model dimensions, the interaction of shocks generated by the model with the tunnel walls must be accurately captured. This implies that proper development of the boundary layer along the tunnel walls, upstream of the signature extraction location and PIV data planes, is crucial for accurate CFD predictions.

Comparisons of the centerline pressure signature predicted using RANS, inviscid, and quasi-one-dimensional analysis is plotted along with experimental measurements from two probe types, and a static pressure measurement at the beginning of the tunnel test-section, in Figures 11 (a) and (b). The two probe types include a $20^{\circ}$ Pinckney probe with smaller length and diameter and a $10^{\circ}$ (included angle) cone probe. Details of the probes can be found in Castner. ${ }^{2}$ Both probes are atypical for sonic boom measurements, which typically have only use $2^{\circ}-4^{\circ}$ cone angles. In Figure 11 (a) it is observed that the RANS analysis predicts the upstream static pressure measurement and the $10^{\circ}$ cone probe data very well. The inviscid analysis tracks the 
quasi-one-dimensional solution as expected, with additional capturing of oblique shocks not accounted for in the one-dimensional analysis. The Pinckney probe appears to measure a similar shape as the $10^{\circ}$ cone probe, but the amplitude of the pressure is approximately 0.08 non-dimensional units below both the RANS and $10^{\circ}$ cone probe data. Since the static pressure measurement at the beginning of the test-section is used to non-dimensionalize the pressure probes, and the test-section is straight, then physically the non-dimensional delta pressure should be close to zero. This is confirmed by the RANS results and $10^{\circ}$ cone probe data, which implies that the Pinkney probe data must be corrected for a reliable comparison. Since data using both probes types is available for all results shown in this work, only the $10^{\circ}$ cone probe data will be used in subsequent comparisons. In Figure 11 (b), a close-up view of the comparison over the streamwise measurement extent is shown. Although the RANS analysis predicts the level of the $10^{\circ}$ cone probe data very well, it does not contain the small wave length features observed in the experimental data. This discrepancy may be measurement error, under-resolution of the tunnel grid, or generated by tunnel wall artifacts (small irregularities) that are not modeled in the CFD geometry. Results from the empty tunnel test illustrate that viscous flow modeling must be utilized to capture the correct flow conditions in the test-section.

\section{III.D. Case 2: Isolated 1.5 inch Chord, $5^{\circ}$ Diamond Wedge in Tunnel}

Before the full configuration was evaluated, the 1.5 inch chord, $5^{\circ}$ diamond wedge grid system was inserted into the tunnel, and CFD analysis was performed. Figures 12 (a) and (b) show a contour plot of Mach number on the symmetry plane using inviscid (top) and viscous RANS (bottom) analyses, respectively. Comparing the two images, it is observed that inviscid analysis predicts a larger incoming Mach number in the upstream portion of the test-section. This larger Mach number is greater than the 1.96 reference value recorded in the experiment. As expected from the theory of oblique shocks, the larger incoming Mach number is causing a smaller oblique shock angle than the RANS analysis predicts. Towards the top center of Figure 12 (b), shock boundary layer interaction effects on the reflected shock from the tunnel wall are captured. These effects cannot be accounted for using inviscid analysis. Shock wave distortion effects are observed in the wake of the diamond wedge and its support structure. These effects influence the trajectory of the slip-line emanating from the trailing edge of the diamond wedge. Differences in the trajectory of the slip-line are observed between the RANS and inviscid analyses. Figures 13 (a) and (b) show a contour plot of pressure on the symmetry plane using both inviscid (top) and viscous RANS (bottom) analyses respectively. The observed upstream pressure obtained using inviscid analysis is lower than the RANS result. RANS analysis predicts an incoming pressure slightly larger that the reference pressure which is consistent with the empty tunnel results. Significant differences in the shock wave patterns between the two analysis methods are observed for three main reasons: incoming flow differences generated in the diverging section of the tunnel, oblique shock/boundary layer effects on the shock reflection angle at the tunnel walls, and wake effects from the diamond wedge and support which distort the shocks through refraction effects.

Figure 14 (a) plots a comparison of the pressure signature predicted using RANS and inviscid analysis with the measured experimental data from the $10^{\circ}$ cone probe. Upstream of the oblique shock generated by the diamond wedge, the RANS and $10^{\circ}$ cone probe data match well, while the inviscid result has significantly lower amplitude. The shock location also matches very well between the probe and RANS result. The inviscid analysis predicts a shock location downstream of the probe data because the oblique shock angle is lower due to the larger incoming Mach number. The amplitude of the shock using RANS with second-order MUSCL reconstruction appears diffuse. In part, this is due to lack of mesh resolution above the diamond wedge (the highly refined plume shear-layer grid fills this volume in the full configuration). In Figure 14 (b) a comparison of the second-order MUSCL and fifth-order WENO reconstructions is shown with the experimental data. The WENO reconstruction greatly improves the amplitude of the leading and trailing edge shocks. This result illustrates the reduction in artificial dissipation using the higher-order reconstruction, even though the overall accuracy remains second-order asymptotically. Excellent comparison between the RANS-WENO5 prediction and the $10^{\circ}$ cone probe data is obtained.

\section{III.E. Case 3: Complete 1.5 inch Chord, $5^{\circ}$ Diamond Wedge and Nozzle in Tunnel}

Steady-state turbulent viscous CFD analysis was performed using the fifth-order WENO reconstruction for the complete configuration. This configuration contains the 1.5 inch chord, $5^{\circ}$ half-angle diamond wedge installed downstream of the 1.7 area ratio nozzle. Five nozzle operating conditions are considered with NPR $=6,8,10,12$, and 14 . Figure 15 shows a contour plot of Mach number on the symmetry plane through the 
model for an NPR $=12$. Oblique shocks are generated by the diamond wedge leading and trailing edges along with an expansion wave created at the midpoint of the diamond wedge. The shocks and expansion wave travel through the jet plume which changes the angle of the shocks caused be a change in the local Mach angle due to the increased Mach number in the jet plume. This correspondingly bends the jet plume upwards relative to the diamond wedge, changing the downstream trajectory of the plume. Observed local increases of the jet Mach number are caused by the decrease in pressure across the expansion wave. Figure 16 (b) shows a contour plot of the pressure on the symmetry plane through the model for an NPR $=6$ along with a line plot of the pressure along the extraction line (shown as a dashed/solid black line in the plot). A strong shock wave downstream of the diamond wedge, approximately four nozzle diameters downstream of the trailing edge is observed. This shock is the coalescence of upstream reflected shocks off the tunnel walls. The reflected shocks pass through the jet plume and interact with the trailing edge shock from the diamond wedge along the signature extraction line.

To begin the validation process of the CFD prediction for the full configuration a comparison of the Schlieren photograph and the CFD magnitude of the density gradient for NPR $=8$ is shown in Figure 17 . Similar shock structures with comparable shock angles are observed. The jet is over-expanded causing a Mach diamond to form upstream of the oblique shock generated by the diamond wedge leading edge. This is observed in both the Schlieren and the computed density gradient magnitude images. The shear-layer of the jet and its interaction with the oblique shocks are more clearly visible in the CFD result. Comparing the time-averaged streamwise velocity from the PIV with the streamwise velocity from the steady RANS computation in Figure 18, streamwise velocity reduction in the shear layer downstream of the nozzle exit is captured along with streamwise velocity recovery (acceleration) throughout the expansion wave interaction zone. Additionally, the shock boundary layer interaction region in the top right corner of the image also matches well, as does the strong reflected shock from the tunnel walls starting at approximately $X=138$ $\mathrm{mm}$ and $Y=-100 \mathrm{~mm}$ in the CFD result. In Figure 19, the vertical component of the velocity is compared between PIV and CFD. Both the interaction of the diamond wedge shocks with the jet and reflections of the shocks generated by the upstream nozzle are consistent. The CFD results appear sharper most likely due to under-resolution of the PIV. In order to compare the turbulent shear stress measured using PIV with a steady-state RANS result, the Boussinesq hypothesis $\tau_{x y}^{R}=-\rho \overline{u^{\prime} v^{\prime}} \approx 2 \mu_{T} \bar{S}_{x y}$, is utilized. Figure 20 plots a comparison between these two quantities. Qualitatively, the turbulent shear matches the shape of the PIV data, but the magnitude of the stress predicted using RANS is much larger than that observed in the PIV. This may be an over-prediction of the turbulent shear stress by the SA turbulence model, or under-resolution of the PIV data. This is the first use of PIV data in the $1 \mathrm{ft} . \times 1 \mathrm{ft}$. SWT and accuracy of the procedure is still under investigation. ${ }^{2}$ Overall good comparisons have been obtained between the CFD with both Schlieren and PIV measurements.

For a more quantitative comparison, the static pressure signature predicted using RANS with the $10^{\circ}$ cone probe data is examined. Figure 21 shows a close-up view of the pressure contour on the symmetry plane for NPR $=6$ over the range of experimental probe locations (shown as the solid black line). The CFD predicted pressure signature and probe measurements are overlayed to illustrate where the shocks captured in the experiment are generated. The first shock is associated with the terminating shock from the top side of the nozzle exit, while the second shock can be traced back to the Mach diamond formed at the first Mach disk inside the supersonic jet. The oblique shock generated by the leading edge of the diamond wedge is the third shock captured in the pressure data, and the final shock is a combination of the terminating shock from the trailing edge of the diamond wedge and the reflected shock from the upper tunnel wall. The static pressure signature predicted using RANS with the $10^{\circ}$ cone probe data are shown for each NPR in Figure 22 (a)-(e). The shock locations between the CFD data and the probe results are very good. Larger peaks are captured in the CFD, but similar amplitudes in the expansion and recovery regions of the $10^{\circ}$ cone probe data are obtained. A flattening of the $10^{\circ}$ cone probe at approximately $X=6.5$ inches is caused by an overpressure maximum related to the device. The over-prediction of the CFD compared to the $10^{\circ}$ cone probe data between $1 \leq X \leq 1.5$ may be caused by model interaction effects with the large probe, and is currently being investigated.

The sensitivity of the deflected oblique shock angles with respect to NPR is illustrated in Figure 22 (f) which plots extracted pressure signature for both the $10^{\circ}$ cone probe measurements (symbols) and the CFD prediction (solid-line). As the NPR increases, the deflected shock angle for the diamond wedge leading edge shock $(3 \leq X \leq 3.5)$ increases along with the upstream shocks generated at the nozzle exit. The diamond wedge trailing edge shock at $X \approx 6.5$ (which interacts with the strong tunnel reflection shock) appears 
relatively stationary with respect to NPR. A comprehensive discussion of the dependence of shock deflection angle with NPR is presented in the next section on jet deflection effects.

\section{Jet Deflection Effects}

In this section the dominant physical mechanism for oblique shock angle deflection by the jet plume will be described. A simple analytic formula will be developed to predict the stream-wise translation of the leading edge oblique shock after it passes through the jet. Accuracy of the formula will be assessed with comparison to the RANS based CFD data presented in the previous section. Figure /reffig:jetDeflDiagram presents a schematic of the jet deflection process. First an oblique shock is generated by the diamond wedge, the angle of this shock, denoted $\beta$, is implicitly defined by the free-stream Mach number and the angle of the wedge such that,

$$
\tan (\theta)=2 \cot (\beta) \frac{M_{\text {free }}^{2} \sin ^{2}(\beta)-1}{M_{\text {free }}^{2}(\gamma+\cos (2 \beta)+2} .
$$

For this test case $M_{\text {free }}=1.96$ and $\beta=38.42^{\circ}$. As the oblique shock travels downstream it interacts with the jet plume, and the local Mach number $M_{\text {jet }}$ of the plume is not equal to $M_{\text {free }}$. Once the oblique shock hits the plume boundary the shock angle is reduced by the difference in Mach angles between the free-stream and the fully expanded jet Mach number,

$$
\begin{aligned}
\beta_{\text {defl }} & =\beta-\Delta \mu \\
& =\beta-\left[\sin ^{-1}\left(\frac{1}{M_{\text {free }}}\right)-\sin ^{-1}\left(\frac{1}{M_{\text {jet }}}\right)\right] .
\end{aligned}
$$

Assuming isentropic flow, the fully expanded jet Mach number is given by,

$$
M_{\mathrm{jet}}=\sqrt{\left(\frac{2}{\gamma-1}\right)\left[\left(\frac{P_{0}}{P}\right)^{\frac{(\gamma-1)}{\gamma}}-1\right]},
$$

where $P_{0} / P$ is the nozzle pressure ratio, which takes value of NPR $=6,8,10,12$, and 14 in this study. Once the oblique shock has reached the opposite side of the plume boundary, and the local Mach number returns to $M_{\text {free }}$, the local Mach angle readjusts and the original oblique shock angle $\beta$ is recovered. Estimating the plume thickness by the diameter of the nozzle $D$ the stream-wise shock translation is given by

$$
\Delta x_{\text {shock }}=D\left[\frac{1}{\tan (\beta-\Delta \mu)}-\frac{1}{\tan (\beta)}\right] .
$$

Moreover, if the shock location for a particular nozzle pressure ratio, say $\mathrm{NPR}_{1}$, is known, then the shock location for a different nozzle pressure ratio, say $\mathrm{NPR}_{2}$, can be estimated by

$$
\Delta x_{\text {shock }}^{\mathrm{NPR}}=D\left[\frac{1}{\tan \left(\beta-\Delta \mu^{\mathrm{NPR}_{2}}\right)}-\frac{1}{\tan \left(\beta-\Delta \mu^{\mathrm{NPR}_{1}}\right)}\right] .
$$

Since tunnel blockage and nozzle support interaction effects change the local Mach number upstream of the diamond wedge in the current configuration, Equation 5 along with the CFD predicted shock location for the NPR $=6$ are used to assess the predictive capabilities of the analytic formula for NPR $=8,10,12$, and 14. To obtain the CFD predicted shock locations, a characteristic line is traced from the leading edge of the diamond wedge to the intersection point with the sensor line, located 1 inch above the nozzle centerline. Figure 24 (a) illustrates the shock tracing procedure for NPR $=14$. Figure 24 (b) shows a plot comparing the stream-wise shock translation from the NPR $=6$ shock location using both CFD and Equation 5 . The trend is matched very well but the analytic formula overpredicts the translation by approximately 0.05 inches over the range of NPRs. This error could be from over-predicting the plume diameter by using the exterior nozzle diameter of $1 \mathrm{inch}$, but this seems unlikely from the images of the plume. Alternatively, the over-prediction equates to an error in Mach angle adjustment $\Delta \mu$ of between 2 and 3 degrees. This is very likely, since the perfectly expanded jet Mach number used in the prediction assumes isentropic flow. While examination of 
the flow-field clearly indicates oblique shocks generated within the plume itself. Overall the prediction is good considering no computational resources are necessary. The prediction also assumes two-dimensionality of the shock/plume interaction, corrections based on a planar shock intersecting a cylindrical plume can be derived to reduce this error if the sensor line is moved further away from the jet centerline.

The jet deflection analysis confirms that local changes in the Mach number are the dominant physical mechanism for the deflection of the oblique shocks as they cross the plume. This is not suprising since all information in supersonic flow, including shocks, must travel along the characteristic lines which are associated with the local Mach angle. The analysis also suggests that exhaust gas properties, such as $\gamma$, which strongly effect the jet Mach number, should be included in simulations of proposed flight demonstrator models. Exhaust gas effects should also be included in future shock/plume interaction wind-tunnel tests to provide CFD validation data.

\section{Summary}

The Launch Ascent and Vehicle Aerodynamics (LAVA) solver framework using structured overlapping grids has been successfully applied to the prediction of oblique shock/plume interaction. A detailed description of the model geometry and generated structured overlapping grid system have been given. RANS CFD analysis has been performed for three geometric configurations: the empty $1 \times 1 \mathrm{SWT}$, an isolated 1.5 inch chord, $5^{\circ}$ half-angle diamond wedge in the tunnel, and the complete model configuration including the 1.5 inch chord, $5^{\circ}$ half-angle diamond wedge and the 1.7 area ratio nozzle operating at five different NPRs. A thorough validation study has been performed including comparisons to static pressure signatures from two different probe types, Schlieren photographs, and PIV measurements. Good comparisons to the $10^{\circ}$ cone probe data have been obtained. A simplified method for predicting oblique shock deflection by a jet plume was developed based on Mach angle deflection by the fully expanded jet Mach number. This analysis confirms that local Mach angle changes caused by the jet plume are the dominant physical mechanism which deflects the oblique shocks.

\section{Acknowledgements}

This work was partially supported by the NASA Fundamental Aeronautics Program Commercial Supersonic Technologies project and by NASA Ames Research Center. The authors would like to thank Raymond Castner of the NASA Glenn Research Center for access to the experimental data and model geometry, Susan Cliff and Donald Durston of NASA Ames Research Center for valuable discussions on shock/plume interaction, and William Chan for many suggestions to improve the overset grids generated for this work. Computer time for this work has been provided by NASA's Advanced Supercomputing (NAS) facility at NASA Ames Research Center.

\section{References}

\footnotetext{
${ }^{1}$ Kiris, C., Barad, M., Housman, J., Sozer, E., Brehm, C., and Moini-Yekta, S., "The LAVA Computational Fluid Dynamics Solver," 52st AIAA Aerospace Sciences Meeting, National Harbor, Maryland, January 13-17 2014, AIAA-2014-0070.

${ }^{2}$ Castner, R., Elmiligui, A., Cliff, S., , and Winski, C., "Plume and Shock Interaction Effects on Sonic Boom in the 1-foot by 1-foot Supersonic Wind Tunnel," 53rd Aerospace Sciences Meeting, Kissimmee, Florida, June 2015.

${ }^{3}$ Genin, F. and Menon, S., "Studies of shock/turbulent shear layer interaction using Large-Eddy Simulation," Computers E Fluids, Vol. 39, 2010, pp. 800-819.

${ }^{4}$ Castner, R., Elmiligui, A., and Cliff, S., "Exhaust Nozzle Plume and Shock Wave Interaction," 51st Aerospace Sciences Meeting, Dallas/Fort Worth, Texas, January 2013, AIAA-2013-0012.

${ }^{5}$ Castner, R., Zaman, K., Fagan, A., and Heath, C., "Wedge Shock and Nozzle Exhaust Plume Interaction in a Supersonic Jet Flow," 52nd Aerospace Sciences Meeting, National Harbor, Maryland, January 2014, AIAA-2014-0232.

${ }^{6}$ Steger, J. and Benek, J., "On the Use of Composite Grid Schemes in Computational Aerodynamics," Technical Memorandum 88372, NASA, 1986.

${ }^{7}$ Moini-Yekta, S., Towards Hybrid Overset Grid Simulations of the Launch Environment, Ph.D. thesis, University of California, Davis, 2013.

${ }^{8}$ Chan, W., Gomez, R., Rogers, S., and Buning, P., "Best Practices in Overset Grid Generation," 32nd AIAA Fluid Dynamics Conference and Exhibit, St. Louis, Missouri, Jun 24-26 2002, AIAA-2002-3191.

${ }^{9}$ Park, M. and Morgenstern, J., "Summary and Statistical Analysis of the First AIAA Sonic Boom Prediction Workshop," 32nd AIAA Applied Aerodynamics Conference, Atlanta, GA, June 2014, AIAA-2014-2006.

${ }^{10}$ Housman, J., Sozer, E., Moini-Yekta, S., and Kiris, C., "LAVA Simulations for the AIAA Sonic Boom Prediction Workshop," 32nd AIAA Applied Aerodynamics Conference, Atlanta, GA, June 2014, AIAA-2014-2008.
} 
${ }^{11}$ Vinokur, M., "Conservation Equations of Gasdynamics in Curvilinear Coordinate Systems," Journal of Computational Physics, Vol. 14, 1974, pp. 105-125.

${ }^{12}$ Spalart, S. and Allmaras, S., "A One-Equation Turbulence Model for Aerodynamic Flows," 30th Aerospace Sciences Meeting and Exhibit, Reno, NV, January 1992, AIAA-92-0439.

${ }^{13}$ Housman, J., Kiris, C., and Hafez, M., "Time-Derivative Preconditioning Methods for Multicomponent Flows - Part I: Riemann Problems," Journal of Applied Mechanics, Vol. 76, No. 2, February 2009.

${ }^{14}$ Housman, J., Kiris, C., and Hafez, M., "Time-Derivative Preconditioning Methods for Multicomponent Flows - Part II: Two-Dimensional Applications," Journal of Applied Mechanics, Vol. 76, No. 3, March 2009.

${ }^{15}$ Van Leer, B., "MUSCL, a new approach to numerical gas dynamics." Computing in Plasma Physics and Astrophysics, Vol. 1, 1976.

${ }^{16}$ Shu, C.-W., "High Order Weighted Essentially Nonoscillatory Schemes for Convection Dominated Problems," SIAM Review, Vol. 51, No. 1, 2009, pp. 92-126.

${ }^{17}$ Nichols, R., Tramel, R., and Buning, P., "Evaluation of Two High-Order Weighted Essentially Nonoscillatory Schemes," AIAA Journal, Vol. 46, 2008, pp. 3090-3102.

${ }^{18}$ Chan, W., "Developments in Strategies and Software Tools for Overset Structured Grid Generation and Connectivity," 20th AIAA Computational Fluid Dynamics Conference, Honolulu, Hawaii, June 2011, AIAA-2011-3051. 
Figure 1. Side view of the $1 \mathrm{ft} . \times 1 \mathrm{ft}$. supersonic wing tunnel (SWT).

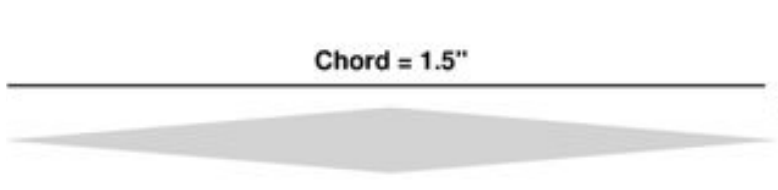

(a)

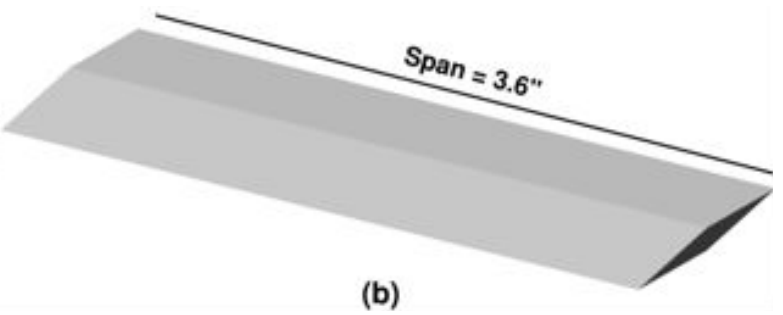

(b)

Figure 2. One and a halg inch $5^{\circ}$ half-angle diamond wedge (a) side view and (b) isometric view.

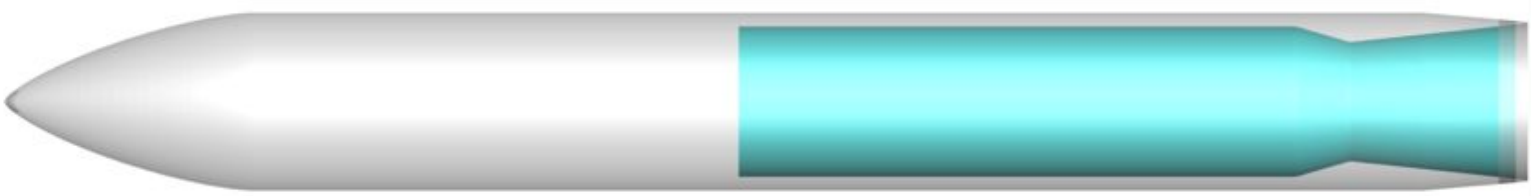

Figure 3. Transparent side-view of the nozzle illustrating the interior and exterior shape.

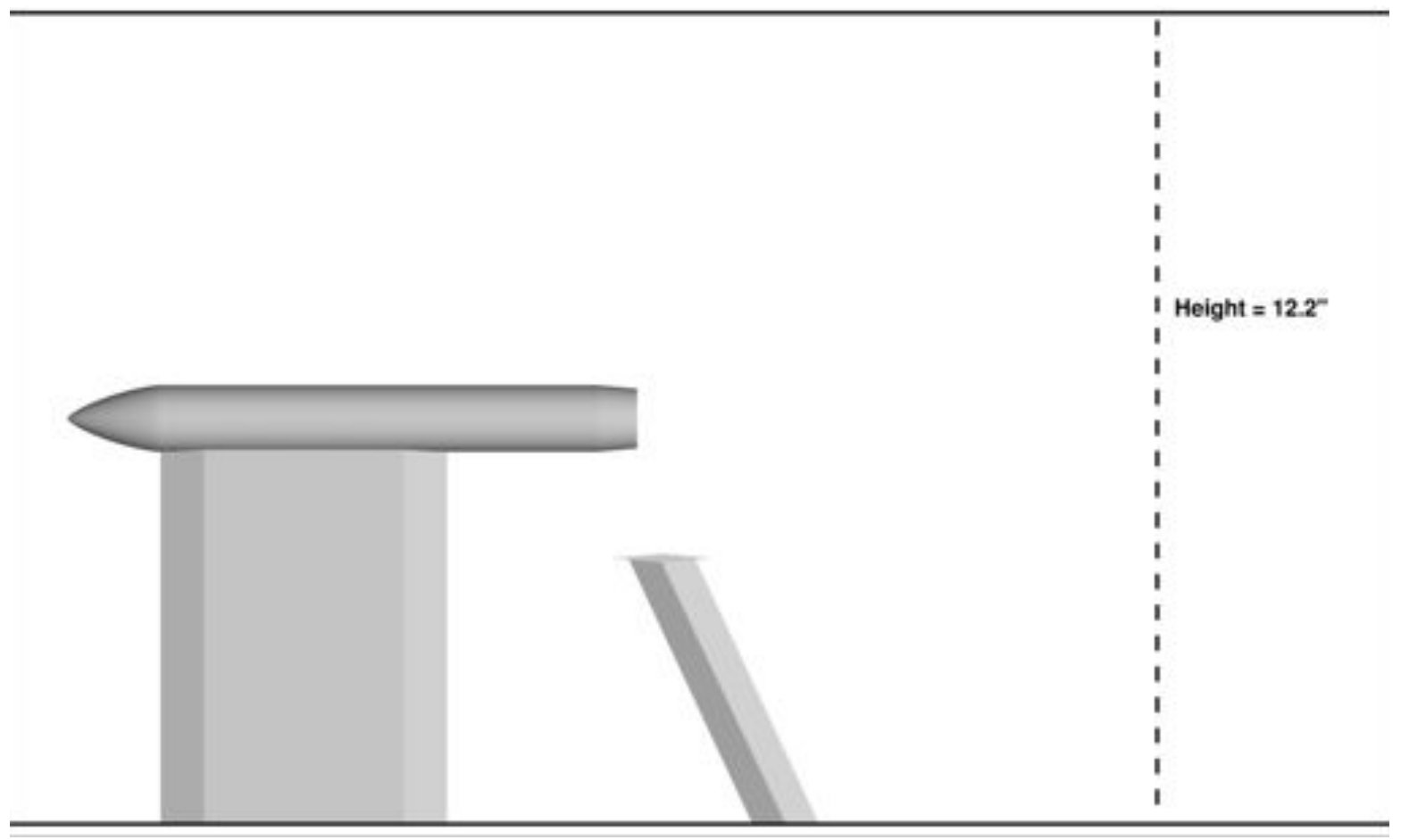

Figure 4. Side-view of the complete installed model configuration containing the 1.5 inch chord $5^{\circ}$ half-angle diamond wedge, the 1.7 area ratio nozzle, and the corresponding support structures. 


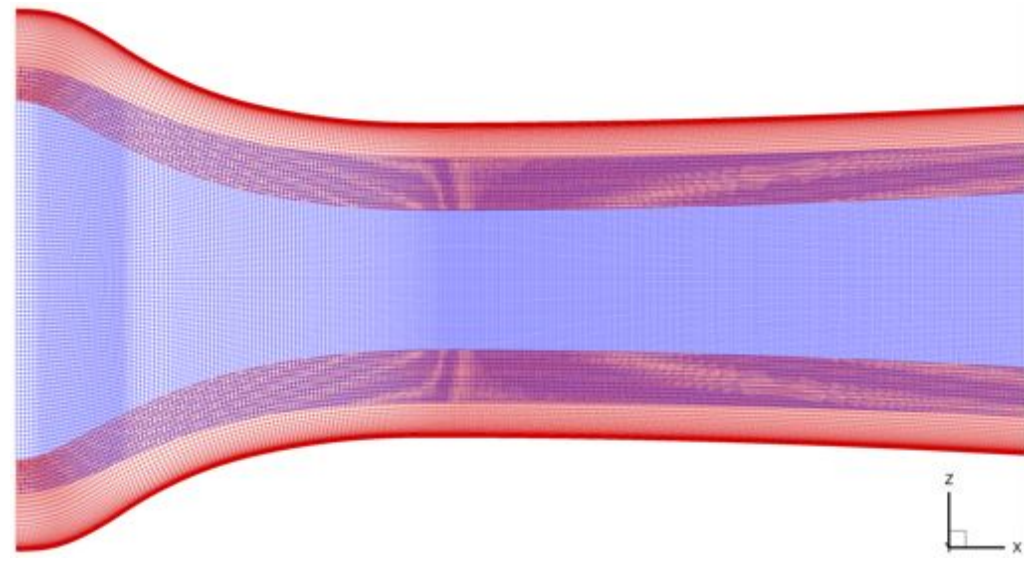

(a)

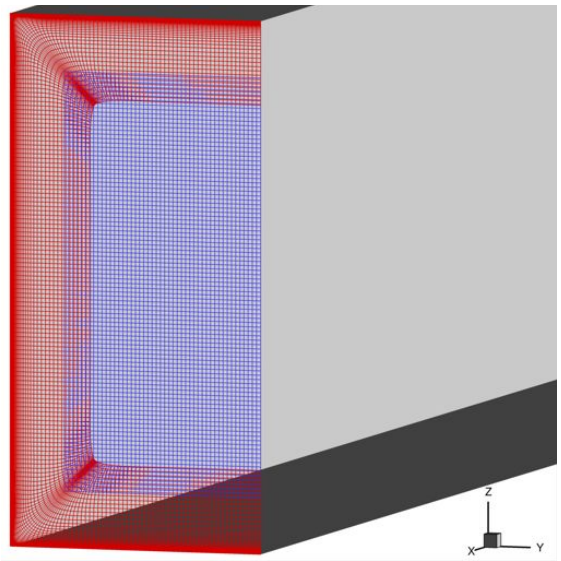

(b)

Figure 5. (a) Side view of the structured overlapping grid system near the converging/diverging section of the tunnel. (b) Structured overlapping grid system of tunnel exit with the solid walls shaded in grey.
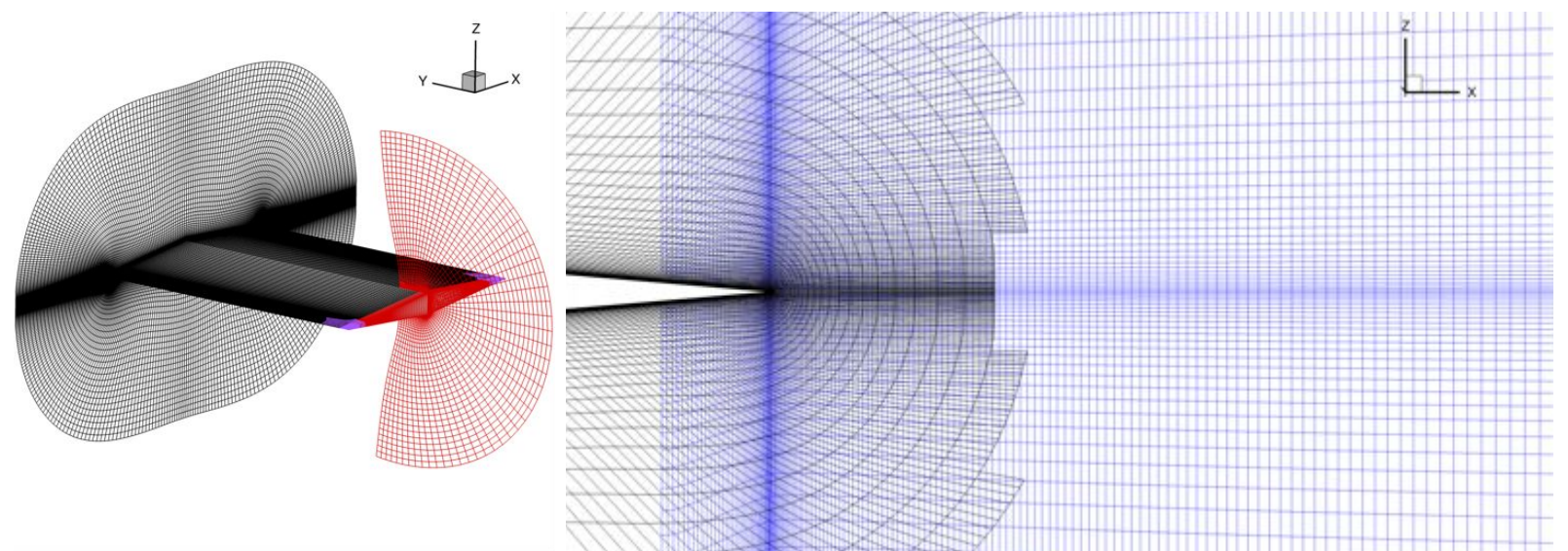

Figure 6. (a) Isometric view of the overlapping grid system for the diamond wedge showing the surface grid, the two-dimensional slice at the symmetry plane, and a slice of the cap grid. (b) Side-view of the diamond wedge/wake grid system showing the transition from the diamond wedge grid to the wake grid.

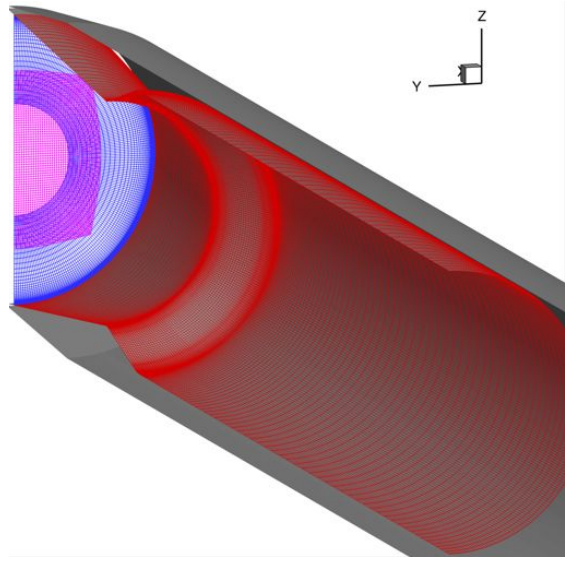

(a)

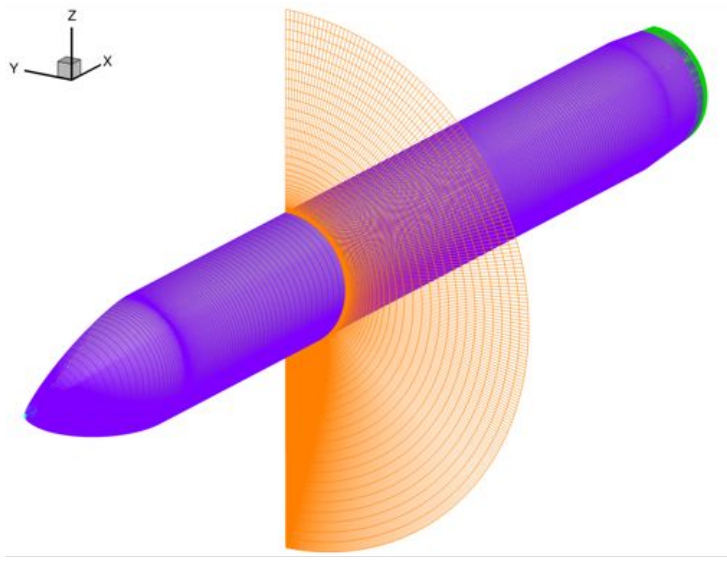

(b)

Figure 7. (a) Surface grid of the nozzle interior along with a slice at the nozzle exit showing the overlap. (b) Exterior nozzle surface grids with a streamwise slice illustrating the clustering used on the bottom half of the nozzle where the nozzle support strut is located 


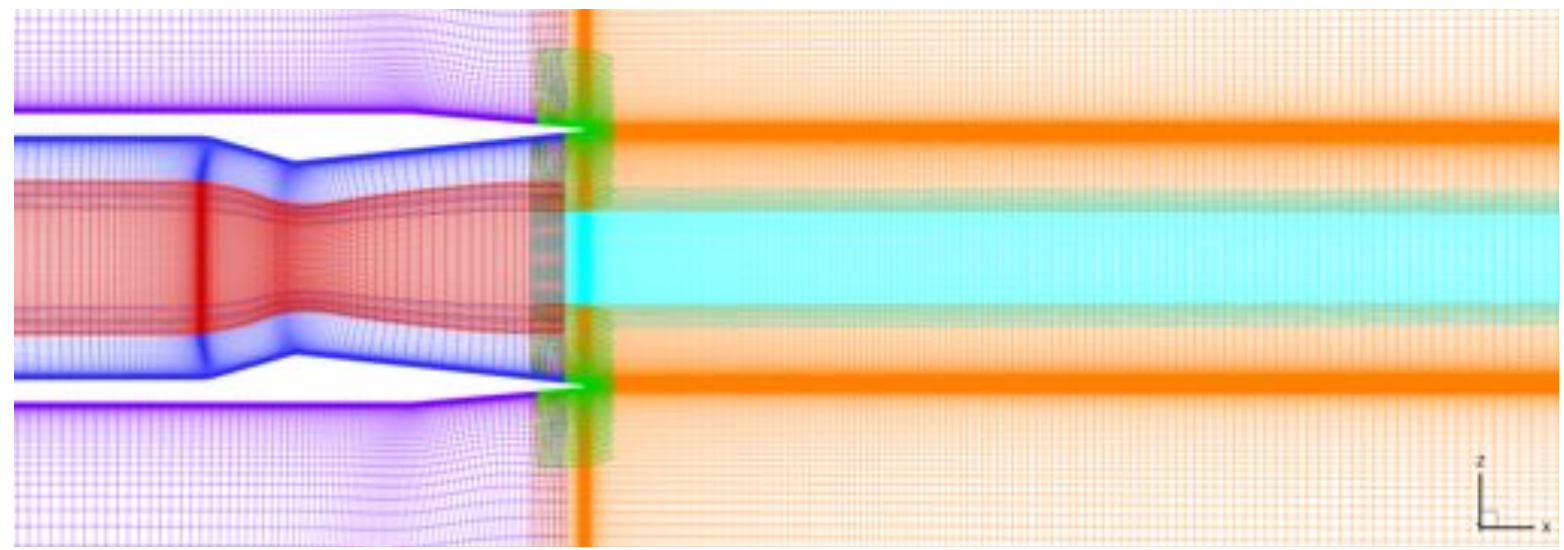

Figure 8. Symmetry plane slice of the overset grid system for the nozzle showing the transition from the nozzle grid to the plume grid.

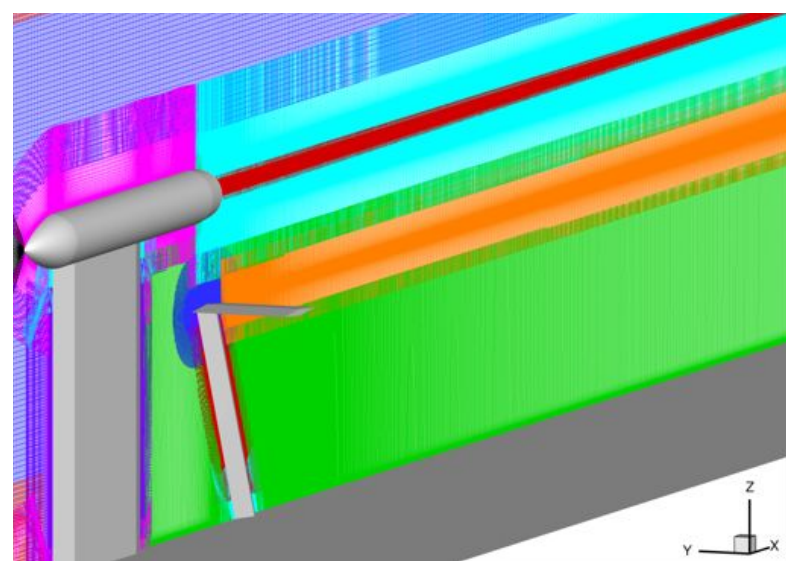

(a)

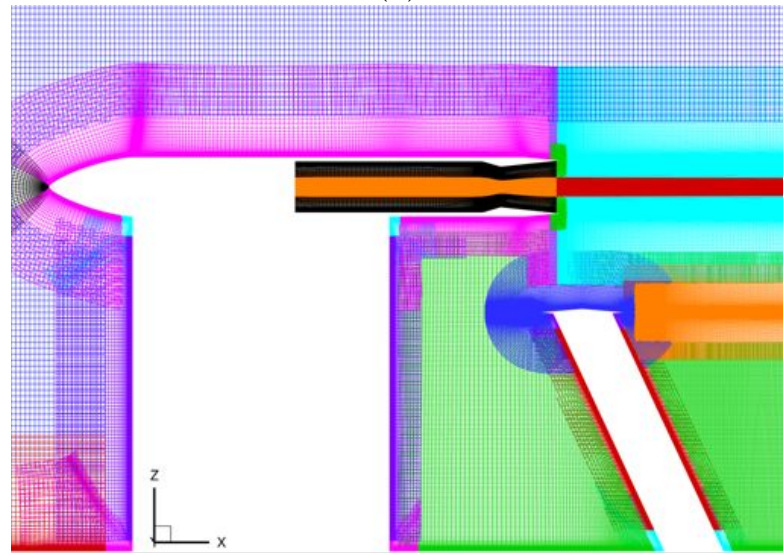

(c)

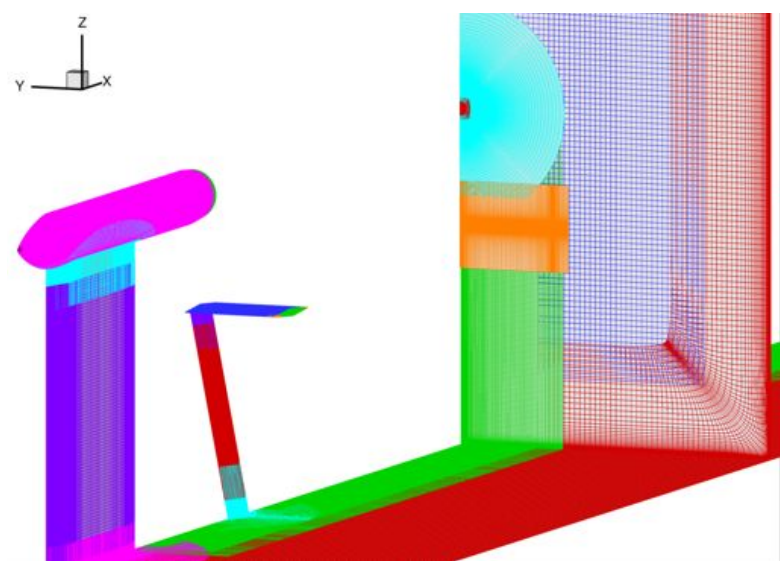

(b)

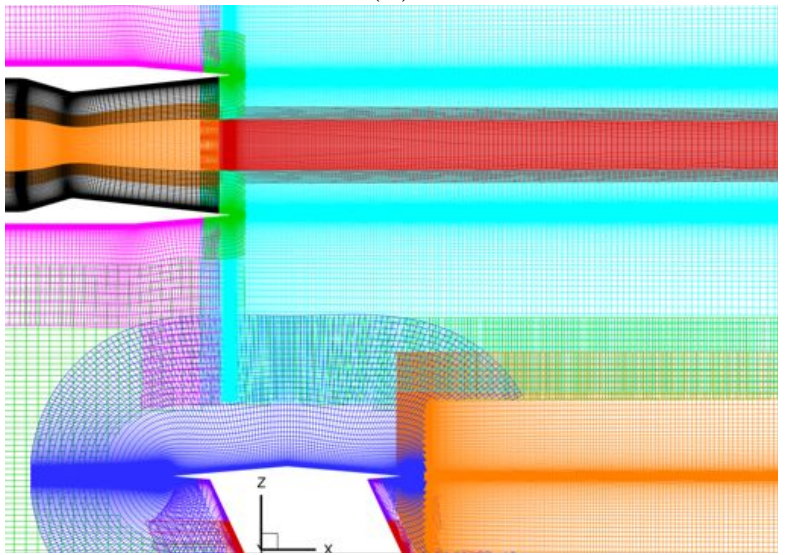

(d)

Figure 9. (a) Isometric view of the installed configuration with overlapping grids on the symmetry plane of the model. (b) Surface grids along with a streamwise slice of the grid system showing the spanwise extent of the plume and wake grids. (c) View of the grid system on the symmetry plane showing the overlapping grid extends of the nozzle, wedge, and support strut grids. (d) Close-up view of the grids on the symmetry plane near the wedge and nozzle exit illustrating the hole-cutting of near-body grids by the anisotropic wake and plume grids. 


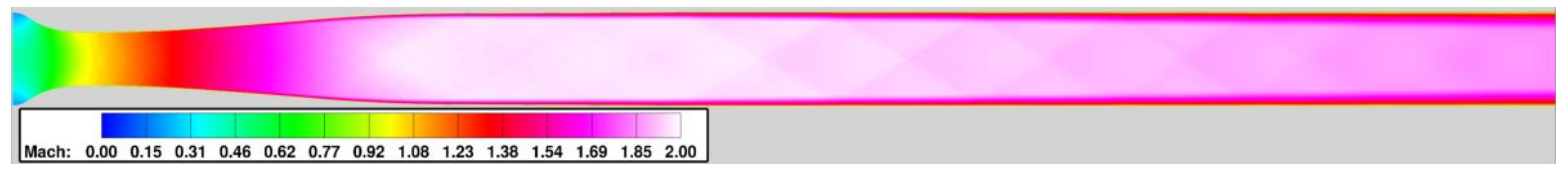

(a)

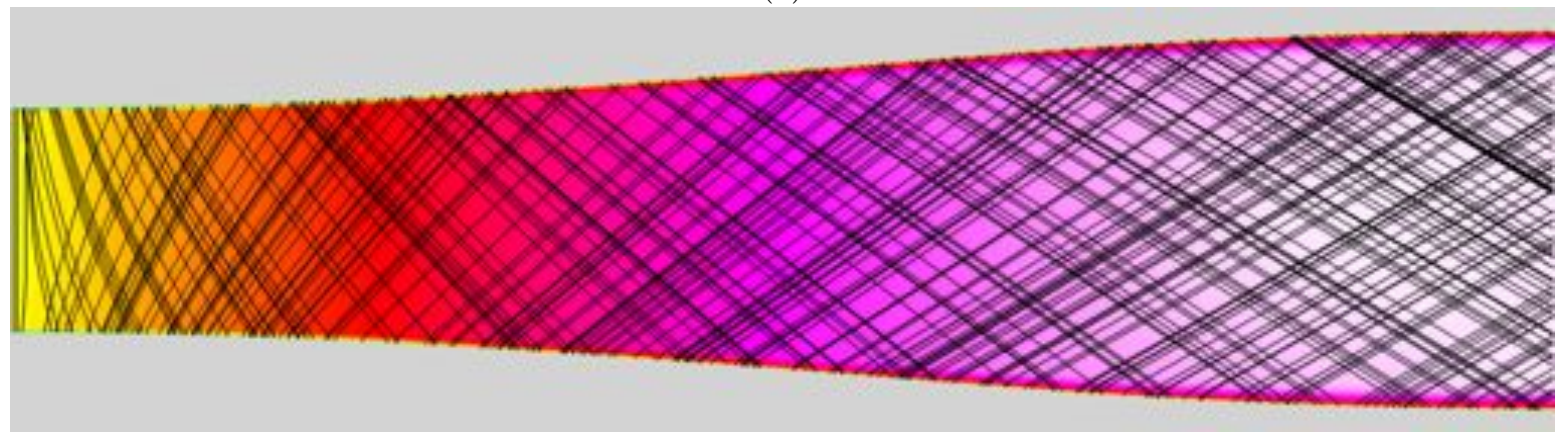

(b)

Figure 10. (a) Contour plot of Mach number on the symmetry plane of the SWT. (b) Close-up view of the diverging section of the SWT showing Mach number on the symmetry plane along with characteristic lines overlayed.

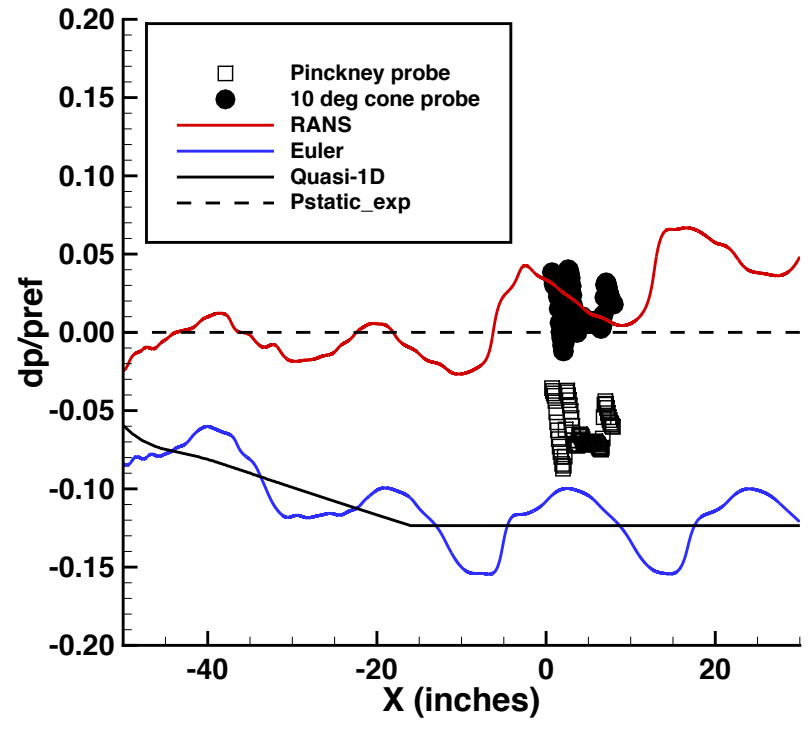

(a)

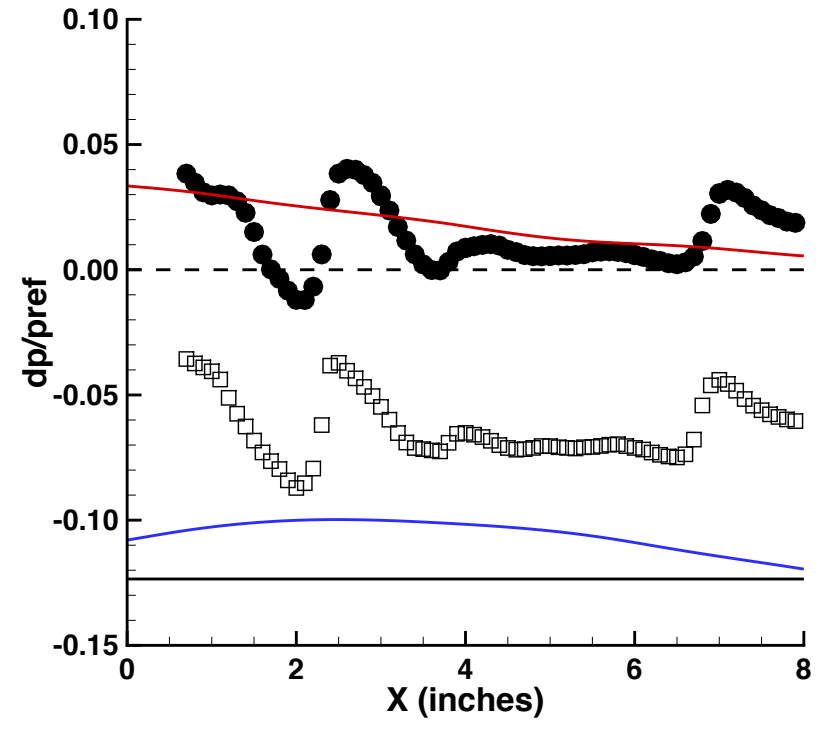

(b)

Figure 11. (a) Comparison of centerline pressure signature in the empty SWT from upstream and through the tunnel test section (experimental data shown in symbols). (b) Close-up view of the centerline pressure comparison. 


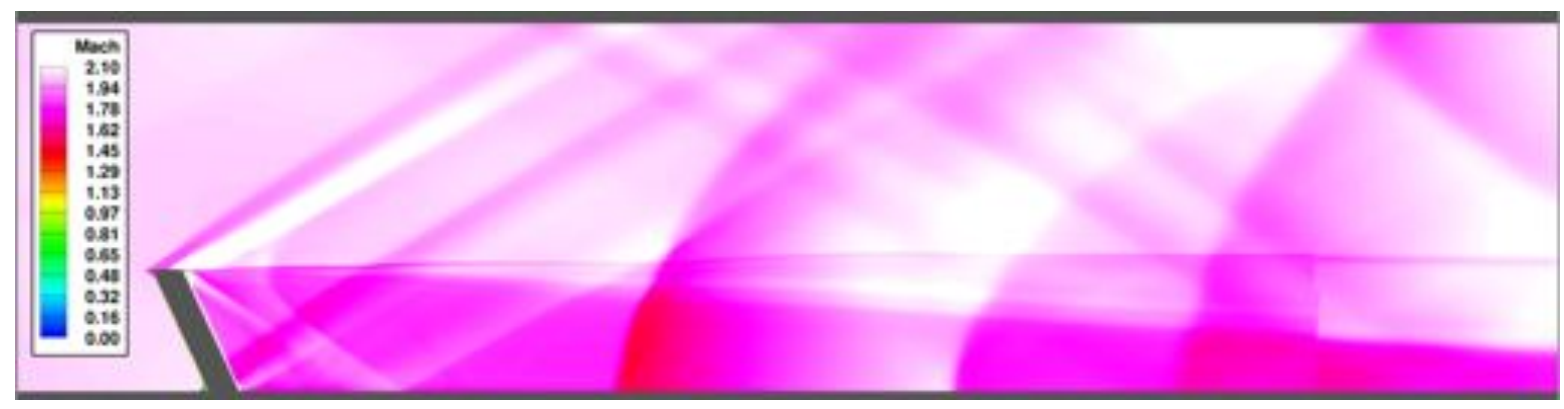

(a)

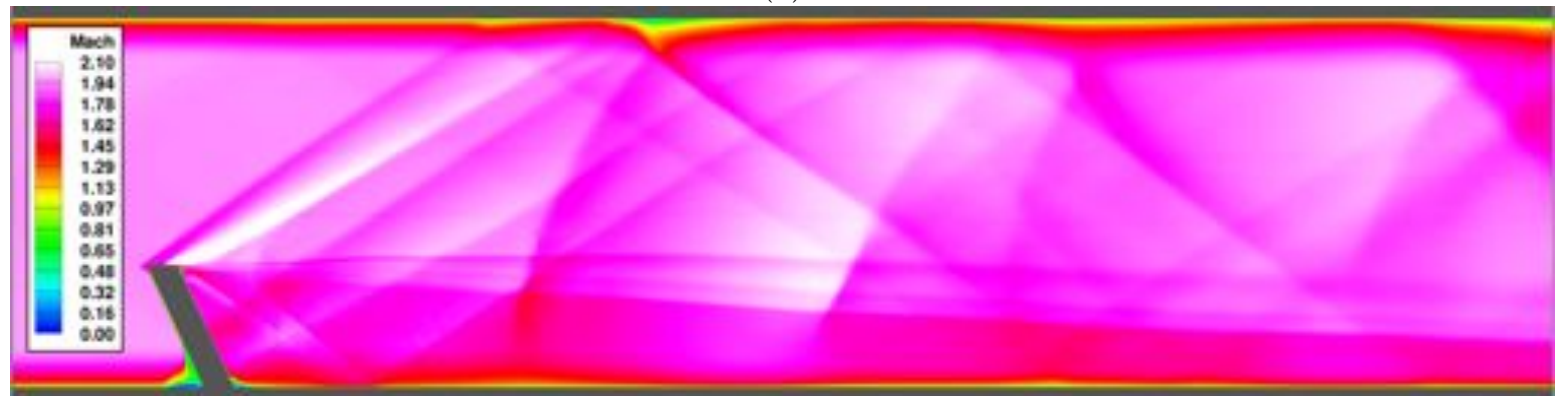

(b)

Figure 12. Contour plot of Mach number on the symmetry plane for the isolated diamond wedge in tunnel configuration using (a) inviscid and (b) RANS CFD analysis.

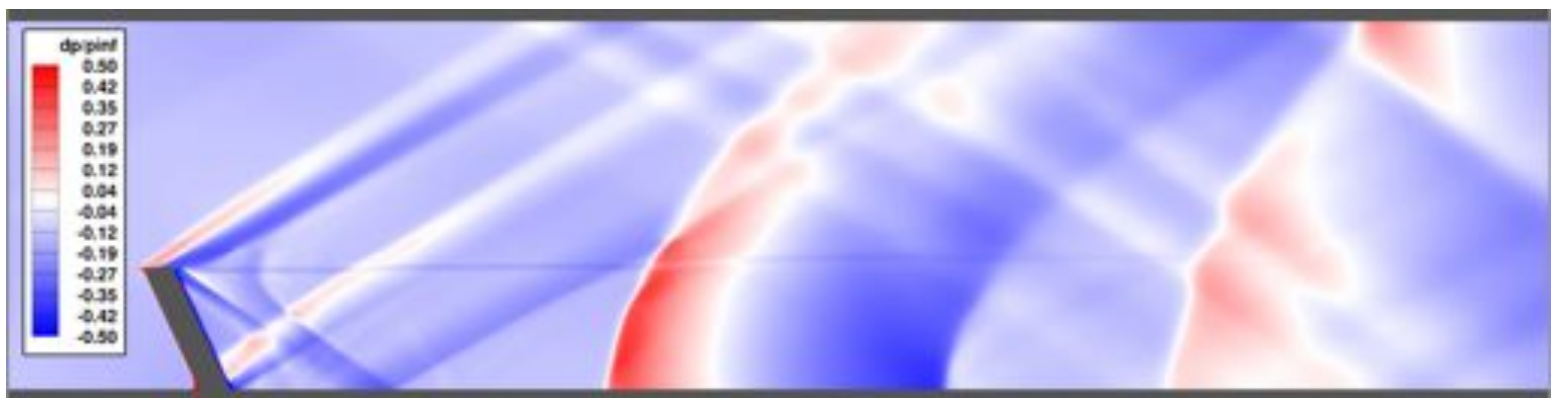

(a)

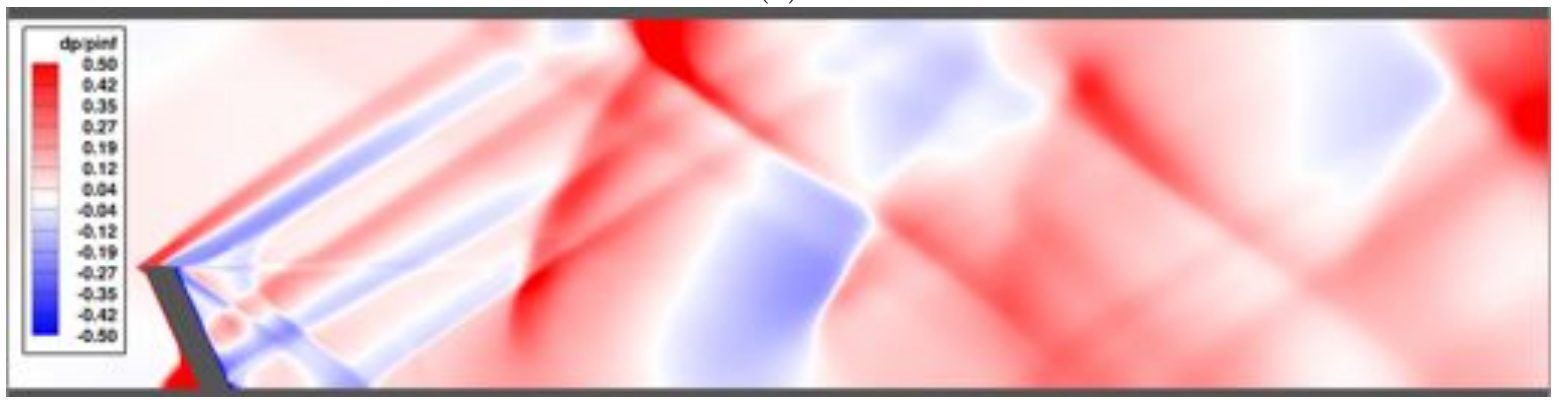

(b)

Figure 13. Contour plot of pressure on the symmetry plane for the isolated diamond wedge in tunnel configuration using (a) inviscid and (b) RANS CFD analysis. 


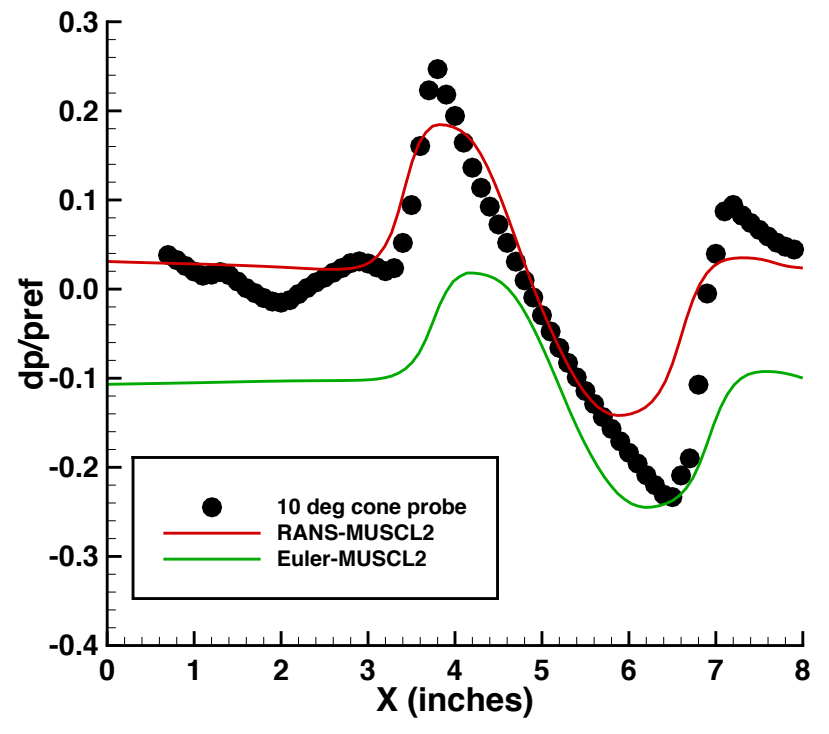

(a)

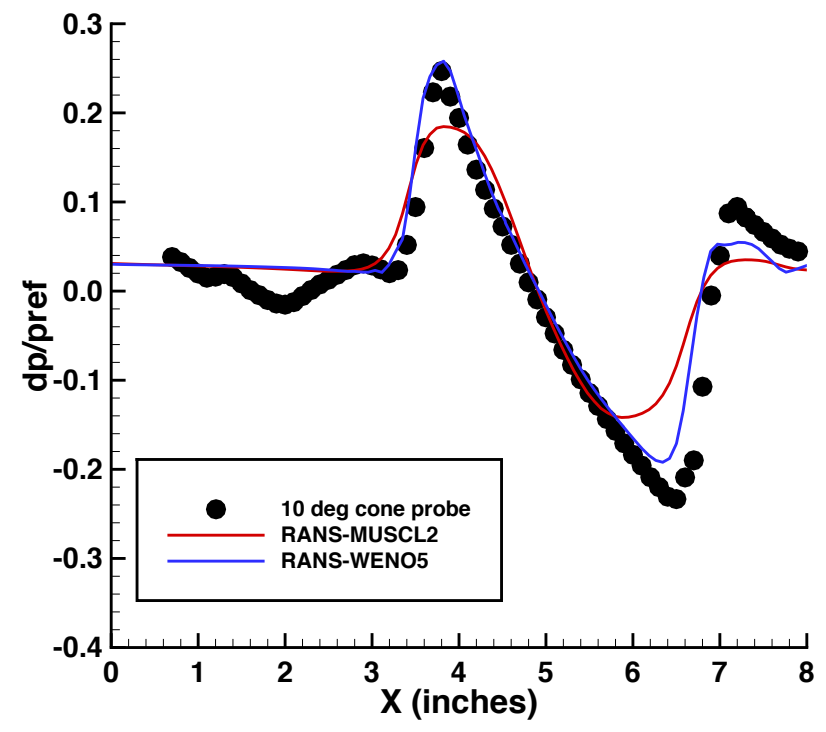

(b)

Figure 14. (a) Comparison of CFD-predicted pressure signature at one inch above the nozzle centerline using RANS and inviscid analysis $10^{\circ}$ cone probe data. (b) Plot of the RANS CFD predicted pressure signature at one inch above the nozzle centerline using MUSCL and WENO reconstruction, illustrating the accuracy improvement obtained using higher-order reconstruction on a fixed mesh. 


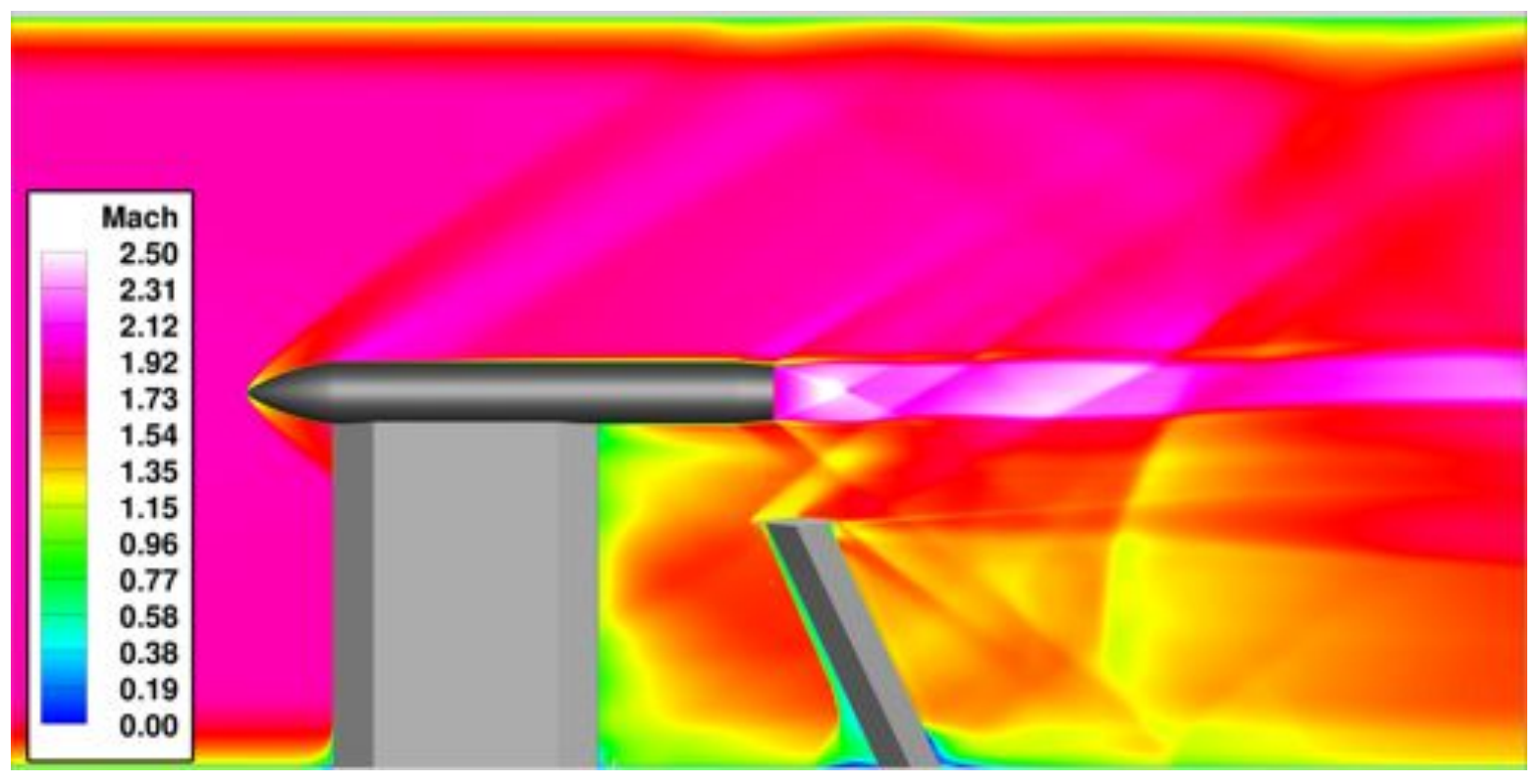

Figure 15. Contour plot of Mach number on symmetry plane for NPR $=12$.

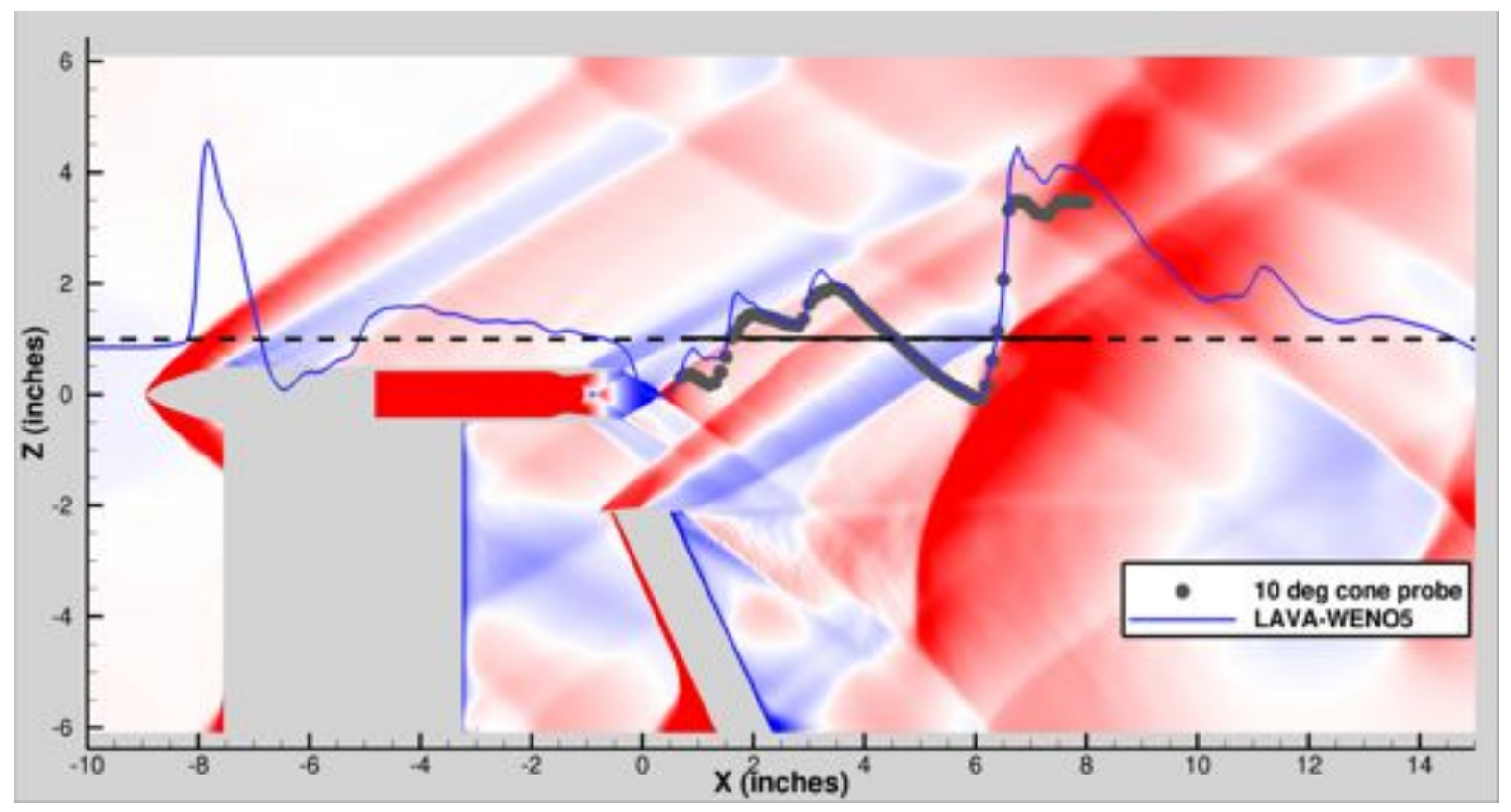

Figure 16. Contour plot of pressure on symmetry plane for NPR $=6$ overlayed with a line plot of pressure along the extraction line shown as a dashed/solid black line. 


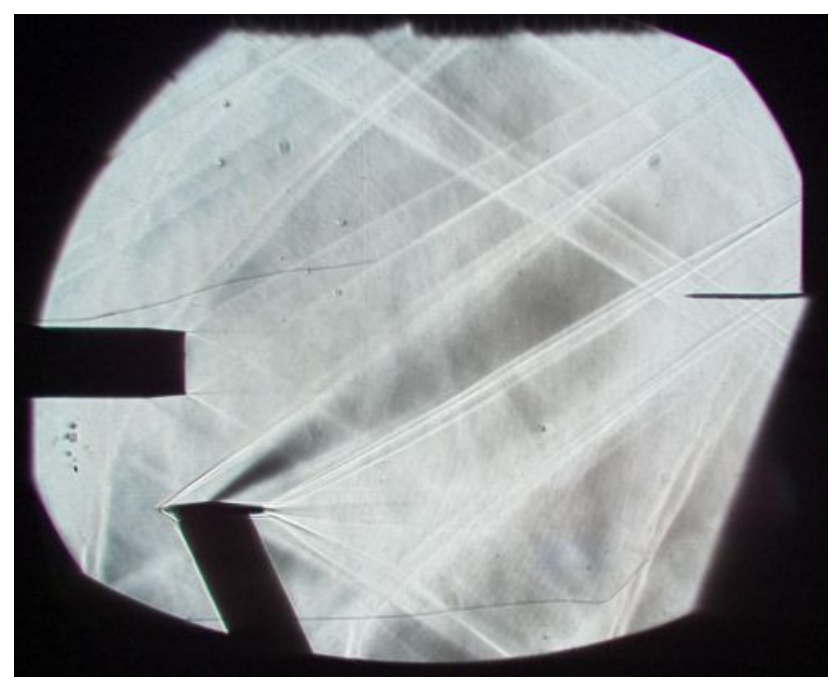

(a)

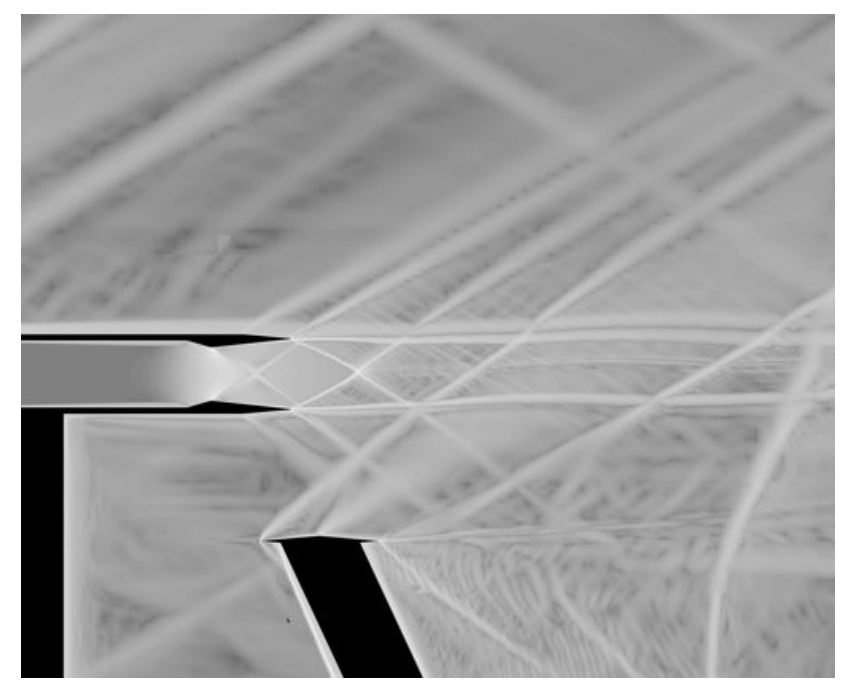

(b)

Figure 17. (a) Schlieren photograph illustrating shock structures on the symmetry plane for NPR $=8$ (from Castner ${ }^{2}$ ). (b) Magnitude of the CFD generated density gradient showing predicted shock structures, shear-layers, and wake effects for $\mathrm{NPR}=8$

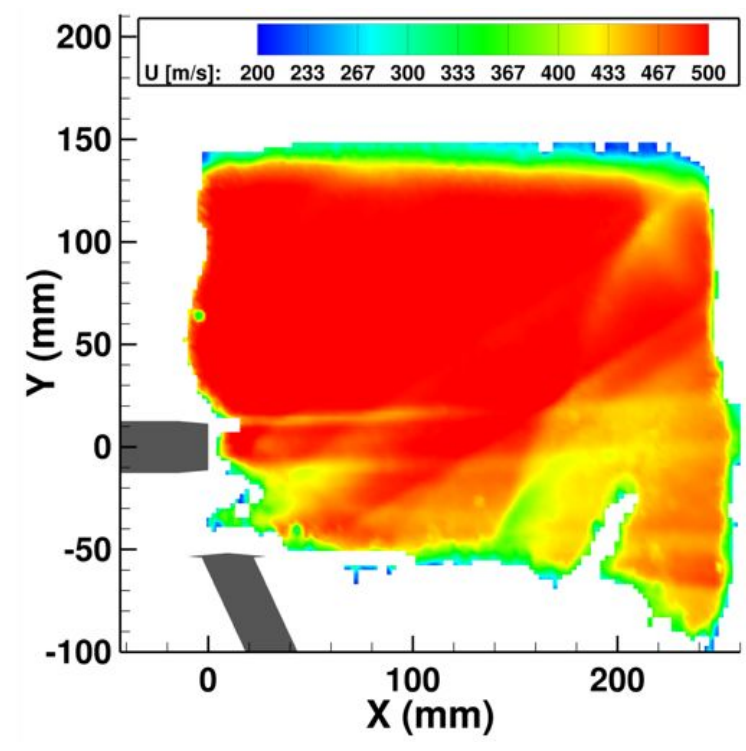

(a)

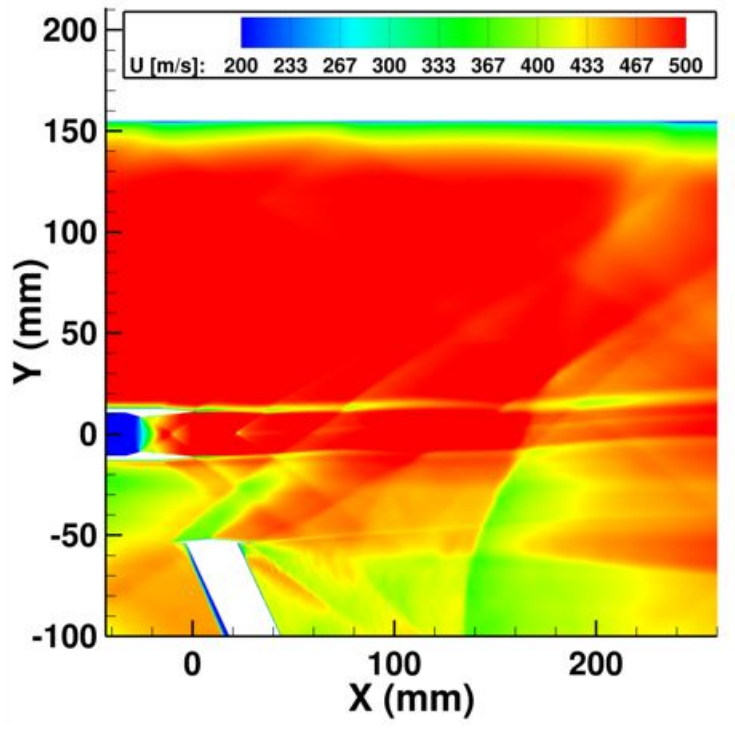

(b)

Figure 18. (a) PIV data of streamwise velocity component for NPR $=8$. (b) Steady-state CFD streamwise velocity component prediction for $\mathrm{NPR}=8$. 


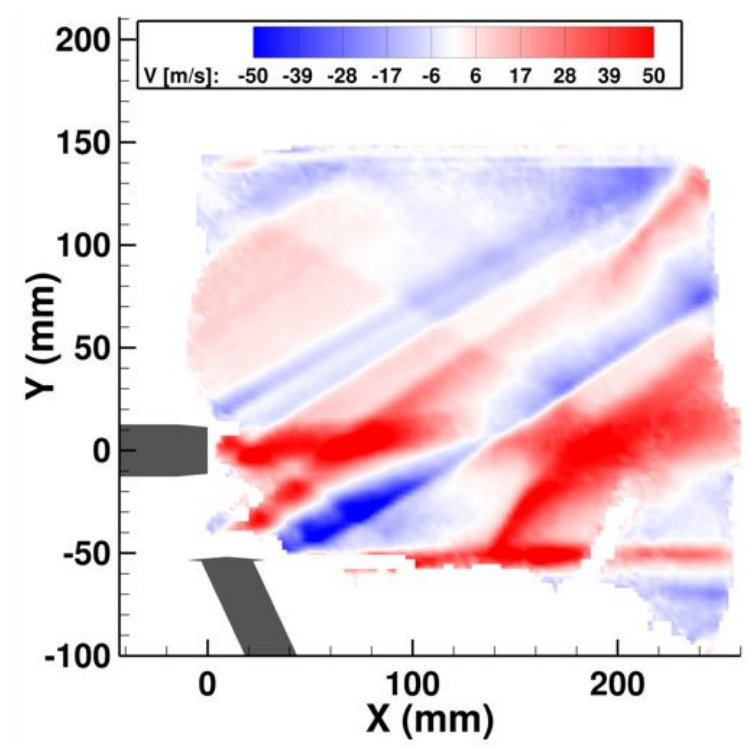

(a)

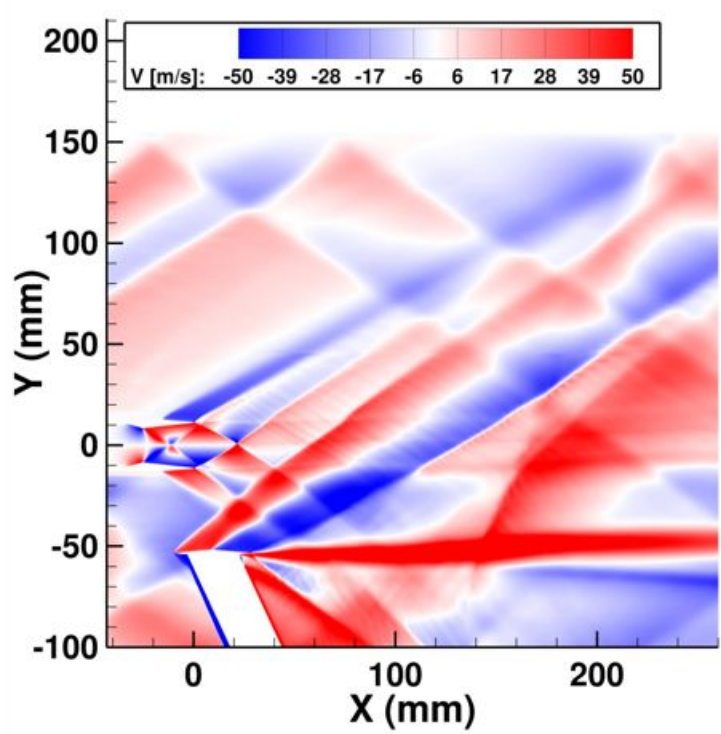

(b)

Figure 19. (a) PIV data of vertical velocity component for NPR $=8$. (b) Steady-state CFD vertical velocity component prediction for $\mathrm{NPR}=8$.

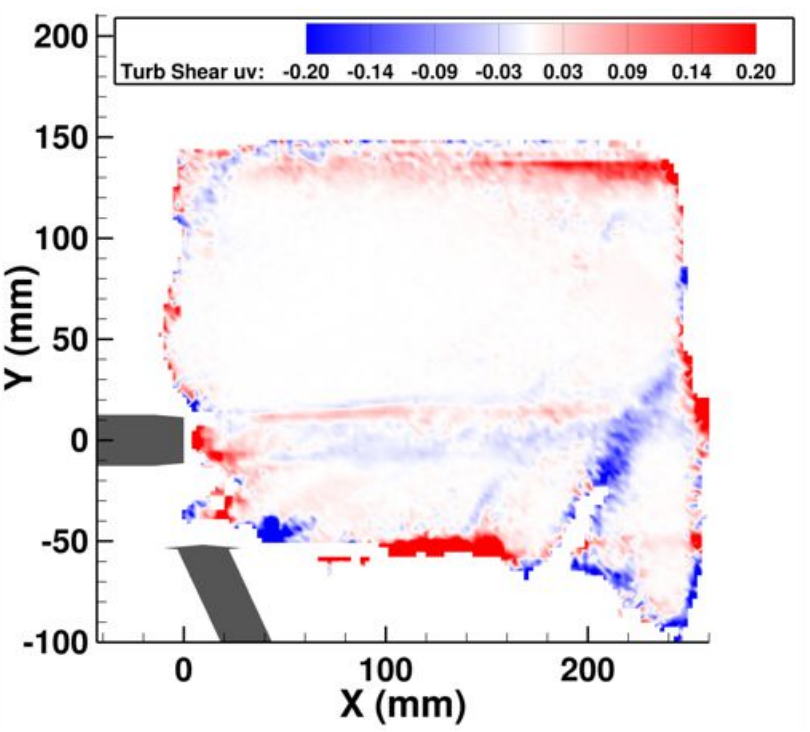

(a)

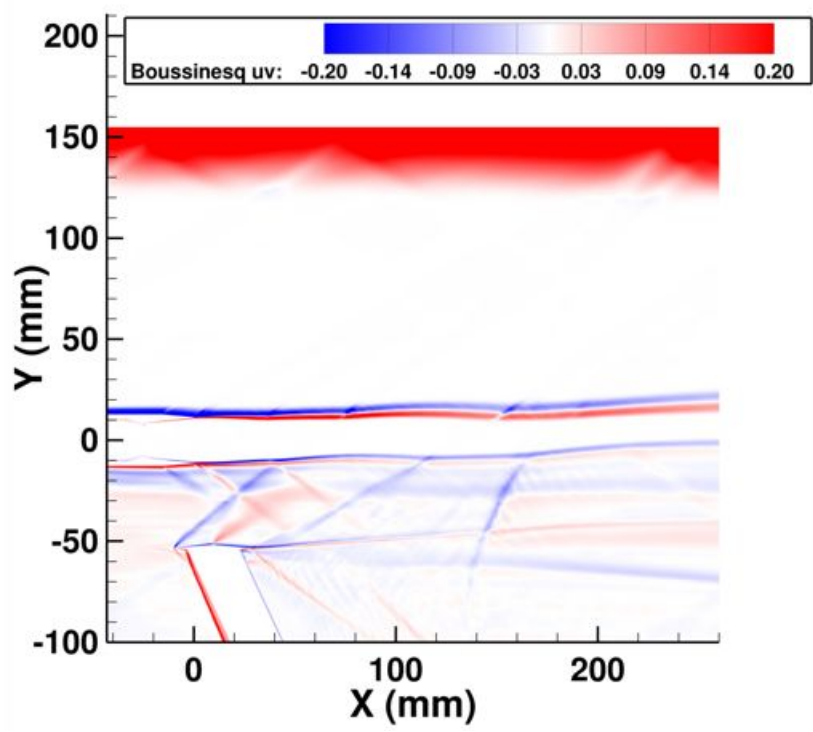

(b)

Figure 20. (a) PIV data of time-averaged turbulent shear-stress for NPR $=8$. (b) Steady-state CFD prediction of turbulent shear-stress using Boussinesq approximation for NPR $=8$. 


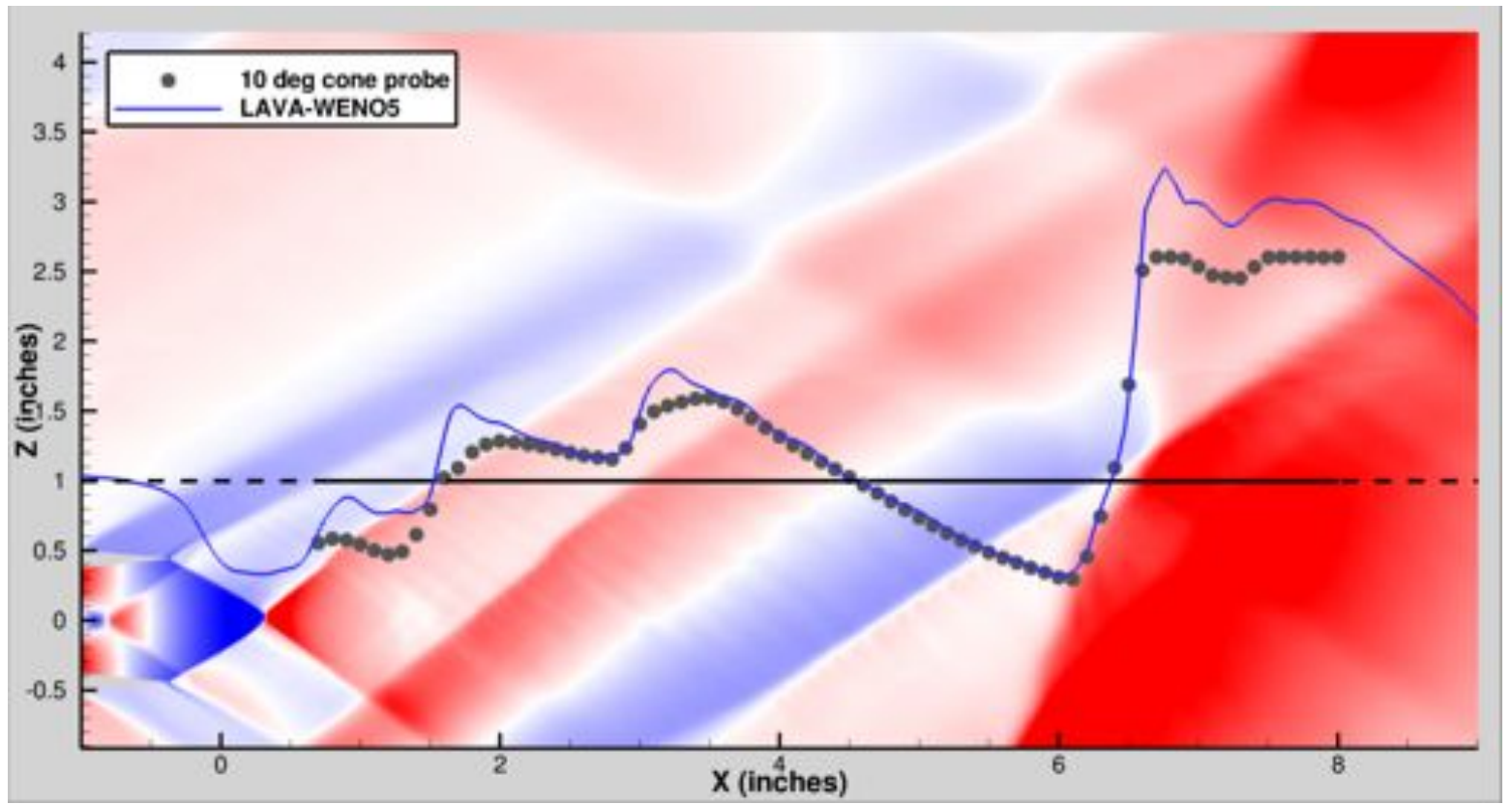

Figure 21. Contour plot of pressure on symmetry plane over the experimental probe range for NPR $=6$ overlayed with a line plot of pressure along the extraction line shown as a dashed/solid black line. 


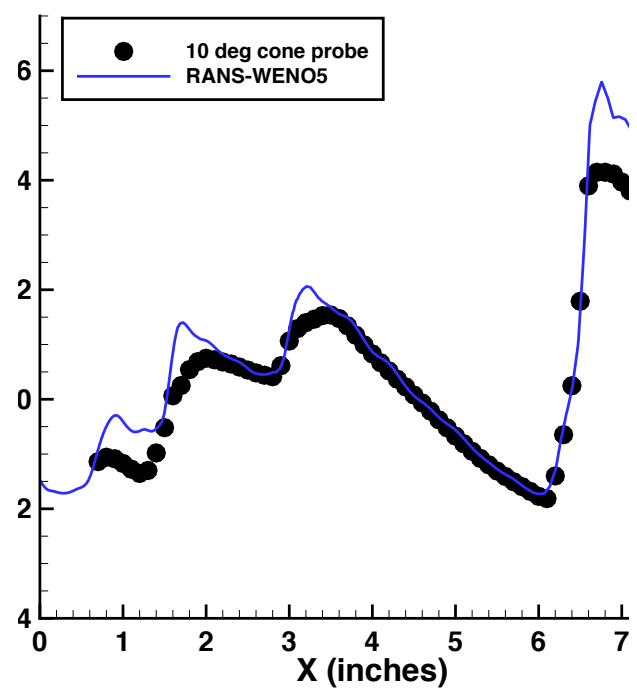

(a) $\mathrm{NPR}=\mathbf{6}$

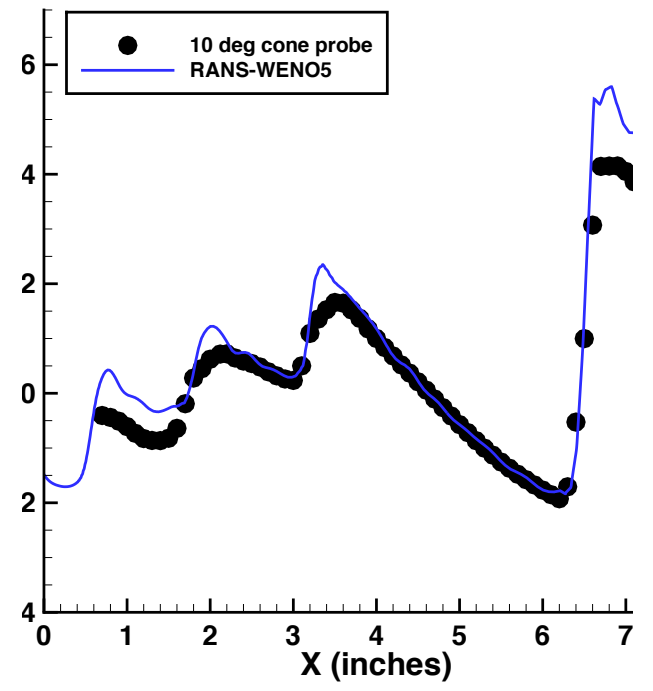

(b) NPR $=8$

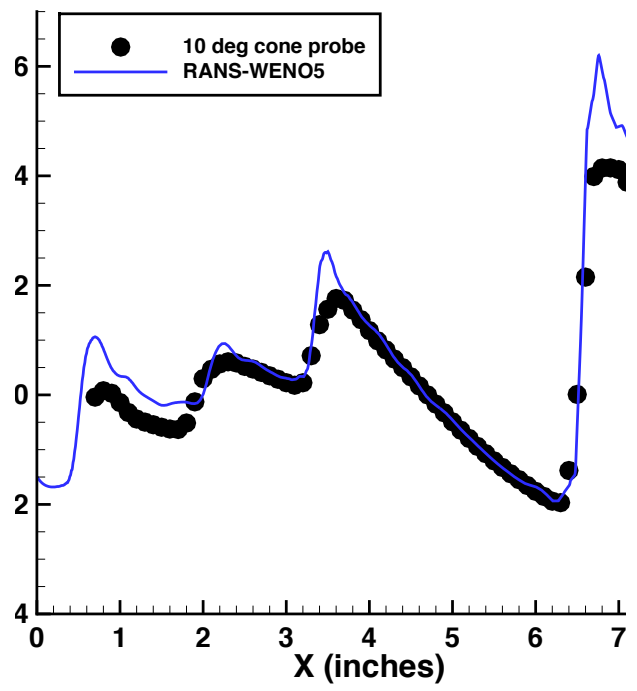

(c) $\mathrm{NPR}=\mathbf{1 0}$

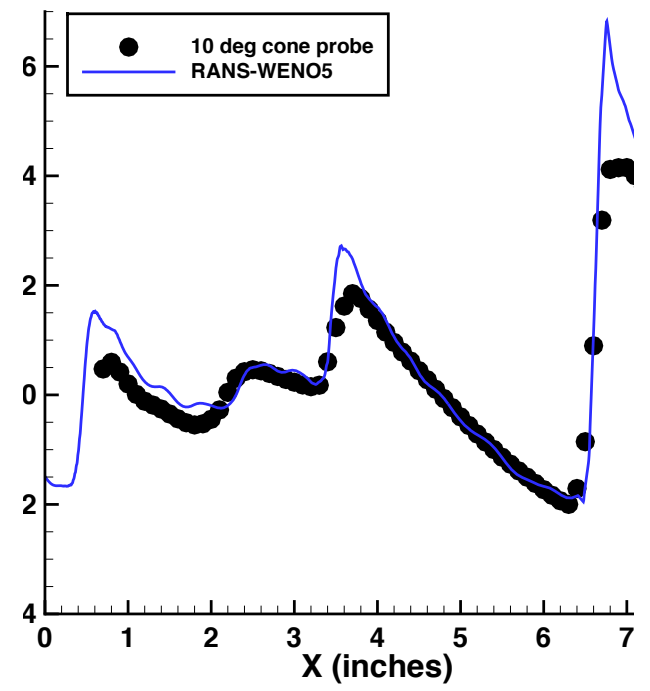

(d) NPR $=12$

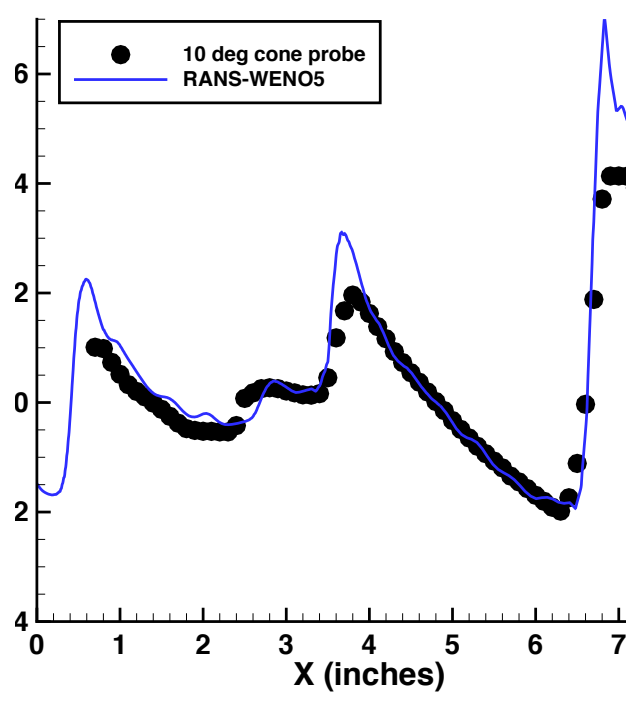

(e) NPR $=14$

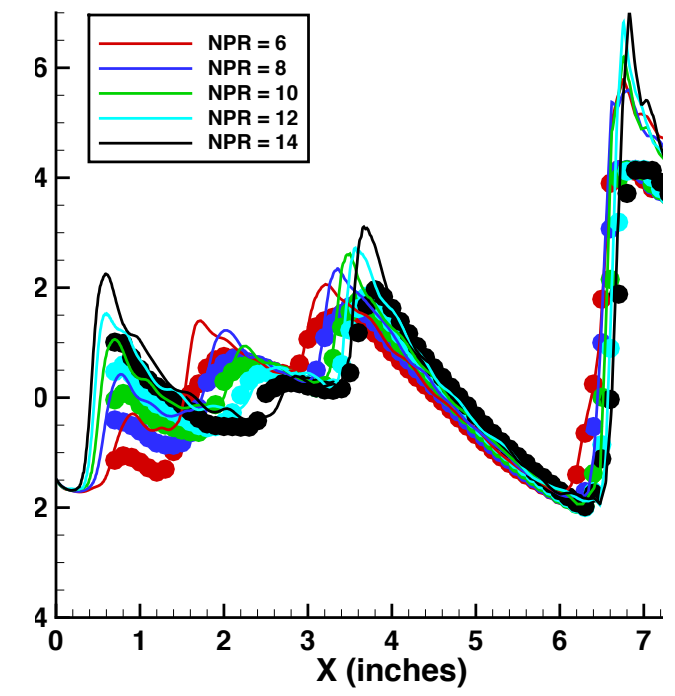

(f) NPR Sensitivity

Figure 22. Comparison of predicted pressure signature with $10^{\circ}$ cone probe data for $(a)$ NPR $=6$, $(b)$ NPR $=8$, (c) NPR $=10,(d)$ NPR $=12$, and (e) NPR $=14$. (f) Pressure signatures illustrating the increased stream-wise translation of the shock with increasing NPR. 


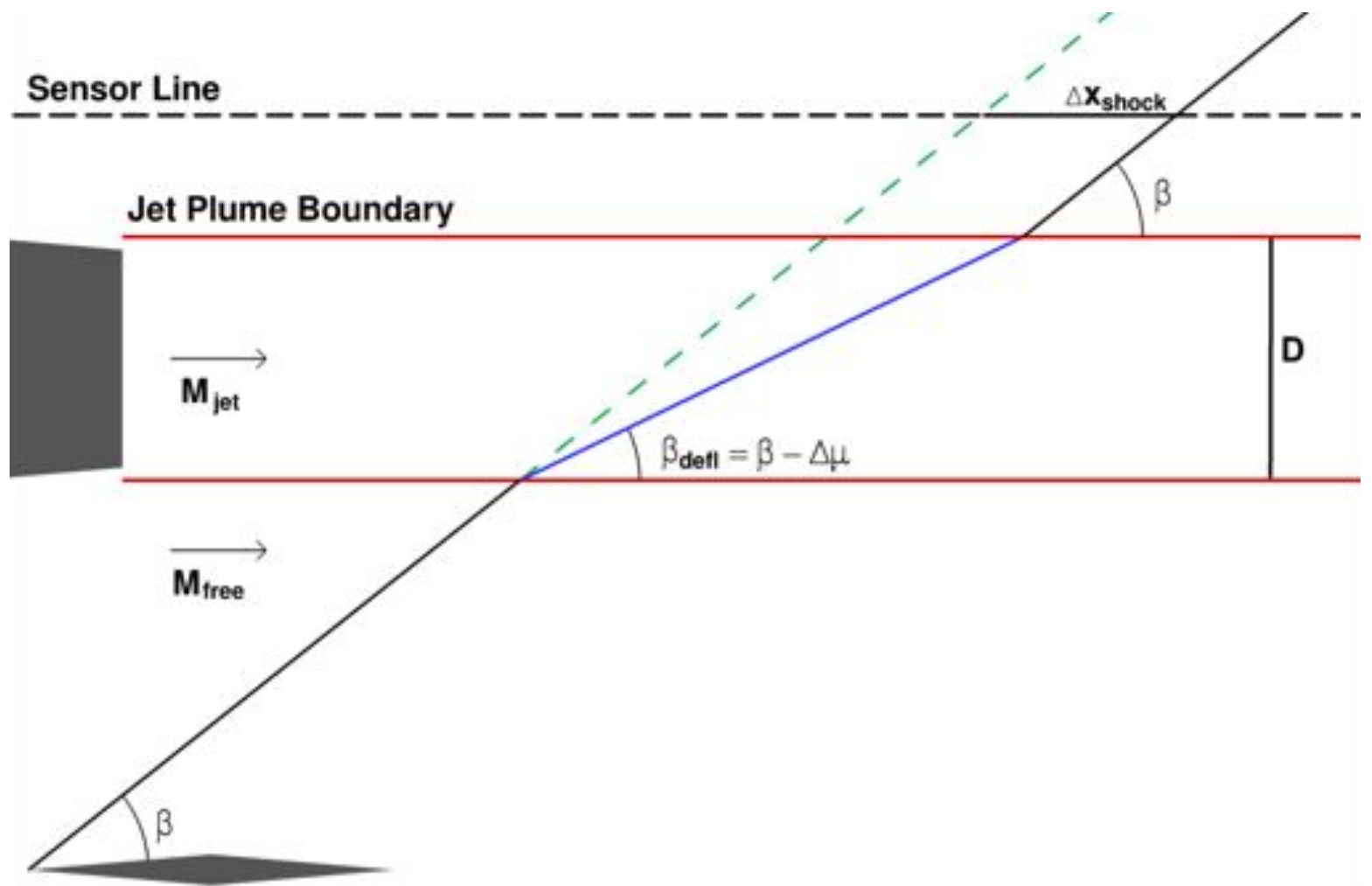

Figure 23. Schematic illustrating the oblique shock deflection effect caused by the jet Mach number, where $\beta$ is the oblique shock angle defined by the free-stream Mach number and the angle of the diamond wedge, and $\Delta \mu$ is the change in Mach angle caused by the difference between the free-stream Mach number and the fully-expanded jet Mach number.

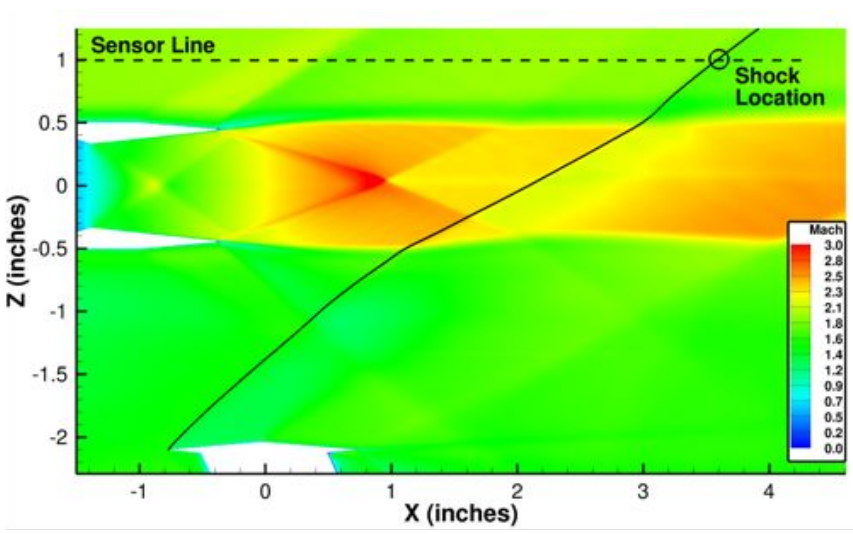

(a)

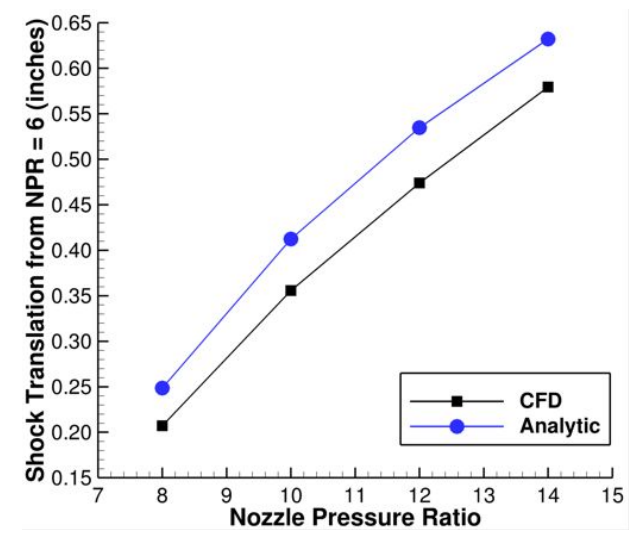

(b)

Figure 24. (a) Contour plot of Mach number on symmetry place for NPR $=14$ along with characteristic trace of the oblique shock generated at the leading edge of the diamond wedge. (b) Comparison of CFD predicted shock translation with analytic formula based on fully expanded jet Mach number deflection. 